Mitteilungen der Österreichischen Geographischen Gesellschaft,

154. Jg. (Jahresband), Wien 2012, S. 261-289

\title{
Auf den Spuren von Oskar Lenz - von Marrakesch ÜBer den Hohen Atlas ins DraA-Tal
}

\author{
Jürgen BitTMAnN, Heuchelheim bei Frankenthal*
}

mit 20 Abb. und 1 Tab. im Text

\section{INHALT}

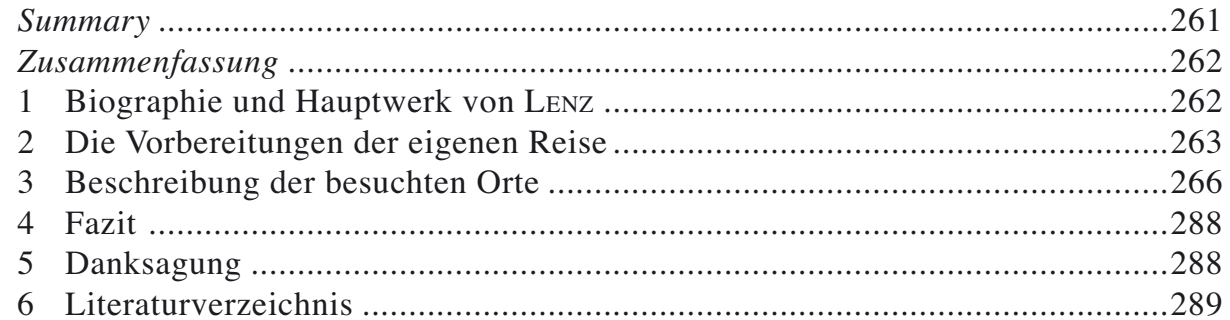

\section{Summary}

On the traces of Oskar Lenz - From Marrakech across the High Atlas to the Draa Valley Based on the travel reports published by Oskar LENZ in 1884, his travel route in Morocco between Marrakech, the passing of the High Atlas Mountains, the crossing of the Anti Atlas and the oasis Foum El Hisn have been reconstructed. To achieve this goal, names of small villages mentioned by him in a temporal sequence have been assigned to places identifiable also today. During a trip in autumn 2011 all identifiable places have been visited and documented. The original texts of Oskar LENZ have been supplemented by own reflections and pictures.

\footnotetext{
* Dipl.-Ing. Jürgen Bittmann, Offsteiner Straße 15, D-67259 Heuchelheim bei Frankenthal; E-Mail: jueba-salam@web.de
} 


\section{Zusammenfassung}

Basierend auf den von Oskar LENZ veröffentlichten Reiseberichten aus dem Jahre 1884 wurde seine damalige Reiseroute in Marokko zwischen der Stadt Marrakesch, dem Übergang über den Hohen Atlas, der Querung des Antiatlas und der Oase Foum El Hisn rekonstruiert. Dazu wurden seine in zeitlicher Abfolge genannten Ortsangaben heute noch identifizierbaren Lokalitäten zugeordnet. Auf einer Reise im Herbst 2011 wurden diese Orte besucht und dokumentiert. Den Originaltexten von LENZ zu den jeweiligen Orten sind eigene Reflexionen und Bilder gegenübergestellt.

\section{Biographie und Hauptwerk von LENZ}

Der Afrikaforscher, Mineraloge und Geologe Dr. Heinrich Oskar Lenz wurde am 13. April 1848 in Leipzig geboren. LeNZ studierte ab 1866 an der Universität Leipzig Mineralogie und Geologie und promovierte dort 1870 zum Dr. phil. mit dem Thema „Über das Auftreten Jurassischer Gebilde in Böhmen“. Anschließend ging er als Lehrer an ein Privatinstitut nach Wien und trat gleichzeitig in die k.k. Geologische Reichsanstalt ein. Im Jahr 1872 erhielt er die österreichische Staatsbürgerschaft.

Von 1874 bis 1877 war LENZ im Dienste der Deutschen Gesellschaft zur Erforschung Äquatorialafrikas in der französischen Kolonie Gabun tätig. Er trat somit im Alter von 26 Jahren seine erste Forschungsreise in Afrika an und bearbeitete bei dieser Expedition die Umgebung des Flusses Ogowa und kartographierte dessen Stromgebiet. LENZ musste diese Expedition aber wegen Krankheit vorzeitig abbrechen. In den Jahren 1877 bis 1879 nahm LENZ an der geologischen Landesaufnahme in Galizien teil.

Bedeutende und große Erfolge in der Erforschung Nordwestafrikas brachte LENZ“ zweite Afrika-Expedition von 1879 bis 1880 . Mit der von LENZ angeführten Expedition im Auftrag der Afrikanischen Gesellschaft in Deutschland durchquerte erstmals ein Europäer die Sahara von Marokko über Timbuktu in den Senegal. Hauptaugenmerk der Reise waren geologische Studien, vor allem die in Nordwestafrika vermuteten Eisenerzvorkommen.

Seine Reise hat er neben Berichten an die Gesellschaft für Erdkunde zu Berlin im Buch "Timbuktu - Reise durch Marokko, die Sahara und den Sudan" (LENZ 1884) ausführlich beschrieben. Neben den geographischen Erkenntnissen ist auch eine Darstellung der staatlichen, sozialen und politischen Verhältnisse Marokkos enthalten. Bemerkenswert ist, dass die ursprüngliche Zielsetzung der Expedition nur die Erforschung Marokkos war. Während der Reise wurde das Vorhaben aber noch um das Ziel Timbuktu erweitert. Somit konnte auch der neue Weg von Norden nach Timbuktu und der Weg von Timbuktu nach dem Senegal erfolgreich begangen werden.

Seine dritte Afrika-Expedition im Auftrag der k.k. geographischen Gesellschaft in Wien unternahm Lenz in den Jahren von 1885 bis 1887 in den neu gegründeten KongoFreistaat - dem Privatbesitz des belgischen Königs Leopold II. Ziel dieser Expedition waren die Erkundung der Handelsverhältnisse im Land, die Kartographierung des Kongoflusses sowie geologische und ethnographische Studien. 
Unmittelbar nach seiner Rückkehr aus Afrika wurde LENZ ordentlicher Professor am Lehrstuhl für Geographie an der deutschen Universität in Prag [Praha]. Hier war er auch Dekan und Rektor, bevor er 1909 in den Ruhestand trat. Lenz starb am 2. März 1925 in Sooß bei Baden in Österreich (ÖAW 1970, S. 140).

Auf der in diesem Beitrag behandelten Marokkoexpedition reiste LENZ bewusst als Einzelreisender mit einem Minimum an Ausrüstung. Er wollte damit den Beweis antreten, größere Erfolge haben zu können als eine vielköpfige, umfangreich ausgerüstete Expedition. Als Tragtiere nutzte er ab Marrakesch zwei Kamele, zwei Pferde, ein Maultier und zwei Esel. Begleitet wurde LENZ von zwei einheimischen Dolmetschern, einem Koch und zwei jungen Dienern. Die Bewaffnung bestand aus einem Gewehr und einigen Revolvern.

LENZ musste sich ab Marrakesch maurisch kleiden und gab sich als türkischer Militärarzt aus Konstantinopel [İstanbul] aus - denn er bewegte sich nun in christenfeindlicher Umgebung. Ein weiterer Garant des Erfolges der Reise war der vom Sultan von Marokko ausgestellte Geleitbrief. Dieser half ihm, die oft feindlich gesinnten Einheimischen zu beschwichtigen, sich unter deren lokalen Schutz zu stellen und deren Ländereien zu durchqueren.

\section{Die Vorbereitungen der eigenen Reise}

Zur Vorbereitung unserer eigenen Marokkoreise haben wir Teile der Reisebeschreibung von Oskar LENZ als Informationsquelle verwendet. Beim Studium der dem Reisewerk beiliegenden Karte kam der Wunsch auf, seine damalige Reiseroute in eine heutige Karte zu übertragen, wenn möglich auf dieser Route selbst zu reisen und nachfolgenden interessierten Reisenden diese historische Route in Marokko zu erschließen.

Aus der Reisekarte von LENZ wurden alle brauchbaren Ortsangaben, wie die von Städten, Orten und Flüssen, in eine Datentabelle übernommen. Jede Information in dieser Tabelle wurde mit einer fortlaufenden Nummer versehen (in der Form: \#xx). Möglichst jede dort beschriebene Lokalität sollte nun mithilfe heutiger gedruckter und digitaler Karten identifiziert werden.

Die Untersuchungen und Zuordnungen sind allerdings auf einen Teil der Marokkoreise von LENz beschränkt. Ausgewählt wurde die Strecke zwischen der Stadt Marrakesch und der Oase Foum el Hisn am Nordrand der Sahara. Dieser Weg war zu Zeiten von LENZ ziemlich unbekannt und gefährlich (Lenz 1884, Bd. 1, S. 260). Die Strecke ist in zwei Teilstrecken unterteilt. Im ersten Teil ist der Weg durch den Hohen Atlas von Marrakesch bis Taroudant beschrieben, im zweiten Teil jener durch den Antiatlas.

Die restliche Reise führte Lenz von Tanger über Fes, Meknes, Rabat und Casablanca [Dar al Beida] nach Marrakesch. Dieser Abschnitt wurde von uns aber nicht berücksichtigt. 


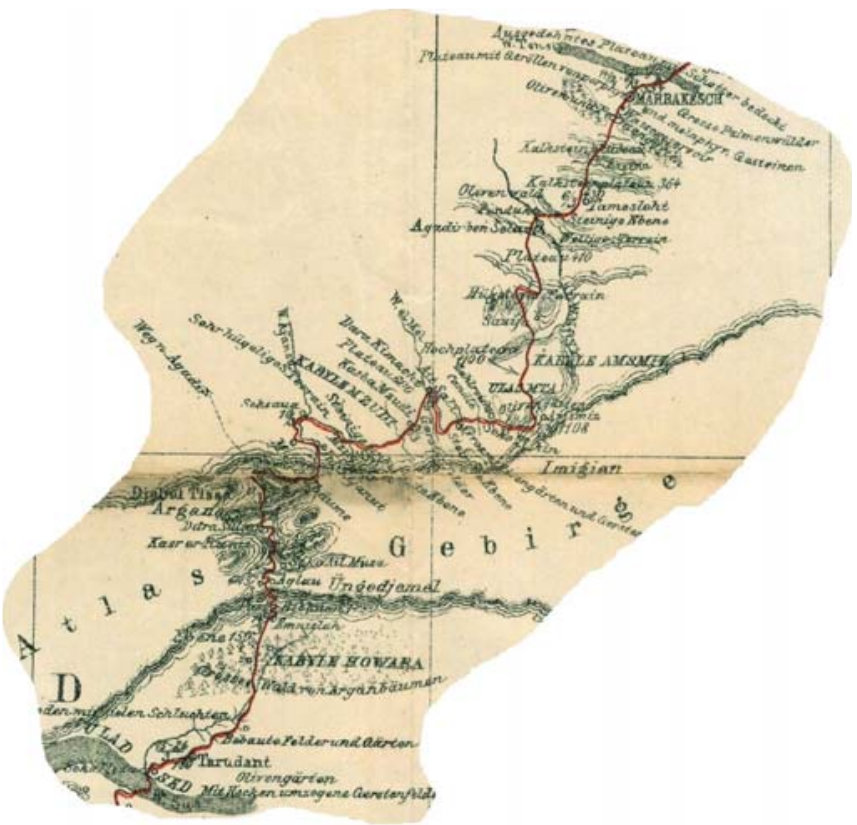

Abb. 1: Kartenausschnitt aus der Reisekarte von LenZ, Abschnitt Marrakesch-Taroudant

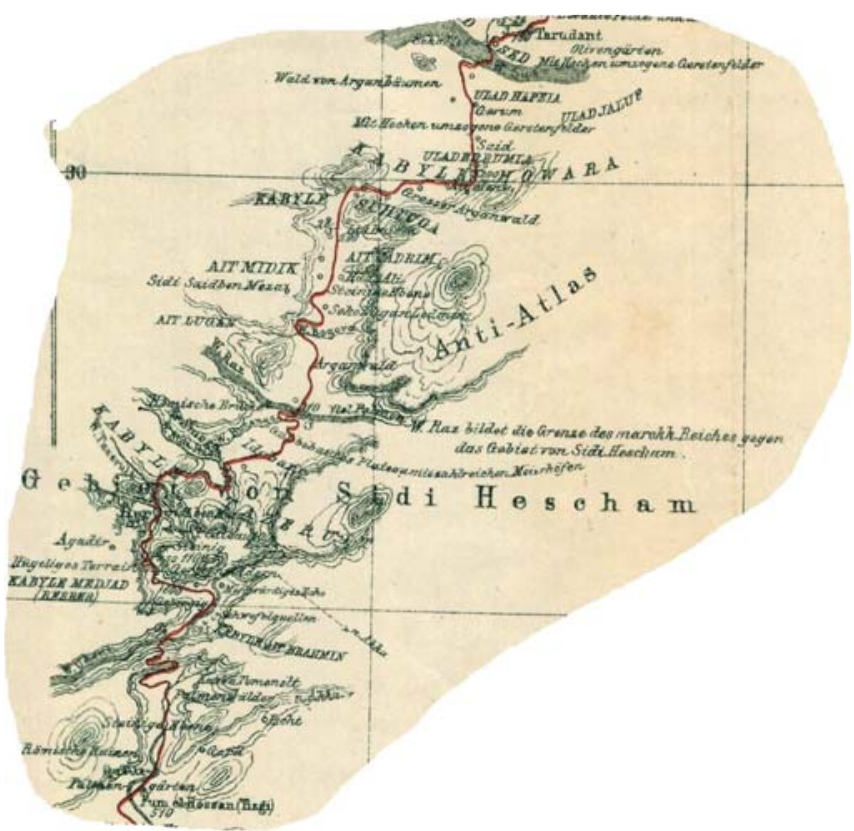

Abb. 2: Kartenausschnitt aus der Reisekarte von LENZ, Abschnitt Taroudant-Foum El Hisn 
Sehr schnell zeigte sich, dass die Ortsangaben in der Reisekarte von LENZ zu wenige Informationen für eine umfassende Rekonstruktion seiner Route boten. Vor allem seine Karte ist sowohl in Ost-West- als auch in Nord-Süd-Richtung stark verzerrt. Richtungsänderungen seiner Reiseroute konnten so nicht auf heutige Karten übertragen werden.

Der von LENZ benutzte Übergang über den Hohen Atlas - einer der interessantesten Abschnitte seiner Reise - konnte so nicht nachvollzogen werden. Die Stadt Taroudant wurde, wie in seiner Karte dargestellt, aus Nordosten erreicht. Alle in dieser Richtung bis zu der am Nordrand des Hohen Atlas gelegenen Stadt Amizmiz gemachten Ortsangaben konnten aber auf modernen Karten nicht gefunden werden. Es musste vielmehr nach weiteren Anhaltspunkten gesucht werden um festzustellen, von wo aus der in südliche Richtung vorgenommene Gebirgsübergang erfolgte.

LENZ hat keine genauen Ortsbestimmungen durchgeführt. Er hat nur eine Wegstreckenaufzeichnung vorgenommen, die Kompassrichtungen und Höhenmessungen notiert. So erklärt sich auch, dass die in seiner Karte eingetragenen Orte bezüglich des verwendeten Koordinatensystems nicht richtig angeordnet sind. Die fehlenden exakten Ortsbestimmungen erklärt LENZ mit folgender Aussage selbst:

„Mein Ritt durch den Atlas nach Tarudant glich eigentlich einer Flucht; ich konnte nur äusserst selten ein Instrument benutzen, ein flüchtiger Blick auf das Aneroid musste gewöhnlich genügen für wichtige Punkte, wo genauere Messungen nöthig gewesen wären, und nur in der Nacht, wenn alles schlief, konnte ich mir die Ereignisse des Tages und die Beobachtungen in die Tagebücher notiren. " (LENz 1884, Bd. 1, S. 284).

Deshalb wurden von ausgewählten Punkten aus der Datentabelle die Entfernungen auf der Reisekarte in Nord- und Westrichtung zum bekannten Standort Amizmiz entnommen und in jeweilige Koordinaten umgerechnet. Dieser Vorgehensweise liegt die Annahme zugrunde, dass Entfernungen und zurückgelegte Strecken von LENz besser dokumentierbar waren als absolute Standorte. Das Ergebnis dieser Auswertung zeigt, dass Lenz die Querung des Hohen Atlas wesentlich weiter westlich startete als es seine Reisekarte vermuten lässt. So konnte die von LENZ genannte Stadt Emmislah am Südrand des Hohen Atlas und der Endpunkt der Gebirgsquerung auf heutigen Karten gefunden werden. Heute ist Emmislah als El Had Menizla (Maroc 1947, Nr. LXI) oder El-Menizla (Michelin 2009, Nr. 742) in Karten zu finden.

Taroudant hat LENZ von Nordwesten her erreicht und nicht von Nordosten. Ein weiterer Beleg dafür ist, dass auch die römischen Ruinen Gaba im Nordwesten von Taroudant in einer heutigen Karte (MARoc 1949, Nr. LII) lokalisiert werden können. Der Einstieg ins Gebirge erfolgte in der Stadt Imintjanut (Lenz 1884, S. 271), nach heutiger Schreibweise Imi-n-Tanout (Michelin 2009, Nr. 742).

Weitere wichtige Informationen in der Datentabelle - wie solche über Landschaftsformen, Siedlungsnamen, eingeschlagene Reiserichtungen und Marschzeiten - wurden aus seinem Reisewerk ergänzend entnommen und chronologisch eingefügt. Somit stehen für den ersten Streckenabschnitt von Marrakesch bis Taroudant die wichtigsten Lokalitäten mit Marrakesch, Amizmiz, Imi-n-Tanout, El-Menizla und Taroudant fest. 
Mit der Lokalität \#22 beschreibt LENZ die Berggruppe des Dschebel Tissa im Hohen Atlas. Diese Berggruppe war aber auch nach intensiver Suche in keiner heutigen Karte zu lokalisieren. Die Erklärung dafür liefert Johannes Justus REIN in einem Vortrag im Jahr 1887, wenn er meint, dass „,der Paß zur Seite des Dschebel Buibaun, Lenz schreibt ,Paß von Bibauan“, nennt ihn auch Dschebel Tisi; doch dürfte hier eine Namensverwechselung vorliegen, da Dschebel auf Arabisch Berg, Tisi im Schellah Paß bedeutet“" (REIN 1887, S. 16).

\section{Beschreibung der besuchten Orte}

Im Folgenden werden die Ortsangaben aus der Beschreibung von Lenz den heutigen Ortsbezeichnungen zugeordnet. Basis sind die chronologisch aufgeführten Nummern aus der Datentabelle. Der Originaltext von Lenz ist kursiv wiedergegeben. Dann sind diesen Orten auch aktuelle Bilder zugeordnet. Aufgelistet sind allerdings nur jene Nummern aus der Datentabelle, die auch lokalisiert und zugeordnet werden konnten.

Ein weiteres Ergebnis dieser Untersuchungen sind die in Satellitenbildkarten von Google Earth dargestellten Routen, die sowohl heutige befestigte Straßen als auch unbefestigte Pisten nutzen. Diese Darstellungen der Google-Earth-Routen liegen in digitaler Form vor und können in heutige Navigationsgeräte übertragen werden. Die Tracks wurden während der Reise noch korrigiert, wenn sich herausstellte, dass vorberechnete Punkte nicht zugänglich oder anfahrbar waren.

\section{\#1 Marrakesch}

„Am 6. März 1880 konnte ich die ehemals so großartige Residenz des marokkanischen Reichs, das gartenreiche Marrakesch El Hamra, verlassen (LENz 1884, S. 260). Nachdem wir den Kranz von Palmenwäldern, welcher Marrakesch von allen Seiten umgibt, verlassen hatten ...". (LENZ 1892, Bd. 1, S. 263)

Das Stadttor, das in südwestlicher Reiserichtung liegt, ist das Bab Aranou. Wir passierten dieses Tor und es ist zu vermuten, dass auch LENZ Marrakesch durch dieses prächtige Tor verließ. Der beschriebene Kranz von Palmenwäldern ist heute nicht mehr vorhanden. Am südlichen Stadtrand dehnen sich weitläufig bebaute Flächen, Olivenhaine und Brachflächen aus. Palmen stehen nur noch vereinzelt.

\section{\#1.1 Steinplatten}

„... betraten wir die mit Steingeröllen bedeckte offene Hochebene; weiterhin kamen kleine Platten von dem schon oft beobachteten horizontal liegenden und in Schalen abgesonderten Kalkstein, die sich bis zu 10 m Höhe über die umliegende Ebene erheben. Die Geröllstücke bestanden vorherrschend aus Eruptivgesteinen." (LENZ 1884, Bd. 1, S. 263) 
Zwischen verdorrten niedrigen Büschen liegen auf der Ebene südwestlich von Marrakesch und in der Richtung der Stadt Tamesloh Kalksteinbrocken. Das Gelände wird teilweise landwirtschaftlich genutzt. Die von LENz beschriebenen bis zu zehn Meter hohen abgesonderten Kalksteine sind nicht mehr sichtbar. Auch auf Suchfahrten abseits der Hauptreiseroute war nichts Derartiges zu finden.

\section{\#2 Wadi Bascha}

„... durchritten dann einige schmale wasserlose Wad, so den Wad Bascha, die dem System des Tensift angehören, ..." (Lenz 1884, Bd. 1, S. 263)

Das wasserlose Flussbett des Wadi Bascha wurde auf einer gut befahrbaren Piste von Ost nach West gequert. Die Flusssohle besteht aus grobem Kies und liegt etwa sechs Meter unterhalb des umgebenden Geländes.

\section{\#3 Tamesloht}

„... und erreichten schon etwas nach 12 Uhr unser heutiges Ziel, das Städtchen Tamesloht. Der Ort ist ganz von Palmen- und Olivengärten eingehüllt, ..." (LENZ 1884, Bd. 1, S. 263)

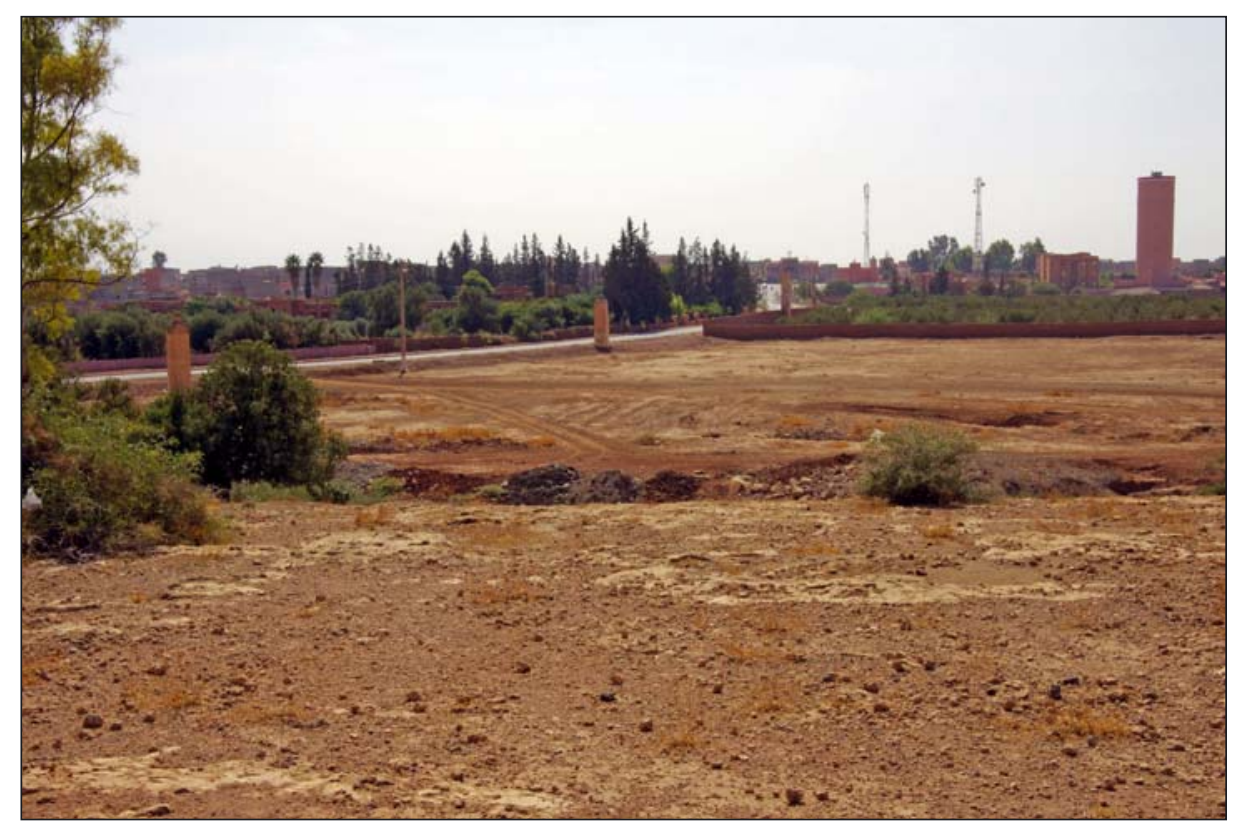

Abb. 3: Tamesloht

Von einer niedrigen Anhöhe aus konnten wir Tamesloht überblicken. Moderne Betonbauten am Ortsrand und alte, niedrige Lehmhäuser im alten Ortskern prägen 
den Ort. Über ihm standen mehrere dunkle Rauchschwaden. Sie stammten von verbrannten Autoreifen, die als Heizmaterial für viele Brennöfen dienen. Tamesloht ist ein Produktionszentrum großer Töpferwaren und gebrannter Fliesen.

\section{\#4 Wadi Nfys}

„Der Weg führte anfangs südwestlich über die steinige Ebene bis zu dem Wad Nfys, der dem Thale Amsmiz entspringend nach Norden fliesst und sich später mit dem Wad Tensift vereinigt und dessen bedeutendster Nebenfluss ist. Das breite Thal des Flusses führt nur einen schmalen Wasserstreifen." (LENZ 1884, Bd. 1, S. 265)

Auf einer steinigen Piste über eine leicht gewellte kahle Ebene fuhren wir von Tamesloht bis zum Fluss Nfys. Dieser führte nur wenig Wasser und eine Querung war problemlos möglich. In den Wassertümpeln tummelten sich Wasserschildkröten. Ob Lenz den Fluss querte oder auf der Ostseite bis Amizmiz weiter wanderte, geht aus seiner Reisebeschreibung nicht klar hervor. Wir jedenfalls folgten der Piste durch den Fluss, um durch freies Gelände bis zum nächsten identifizierten Punkt der Reise zu kommen.

\section{\#6 Tonschiefer}

„Der Fluss durchbricht hier ein hügeliges, nicht leicht zu passirendes Terrain; anstehend bemerkte ich bläulichen Thonschiefer fast senkrecht stehend. "(LENZ 1884, Bd. 1, S. 265)

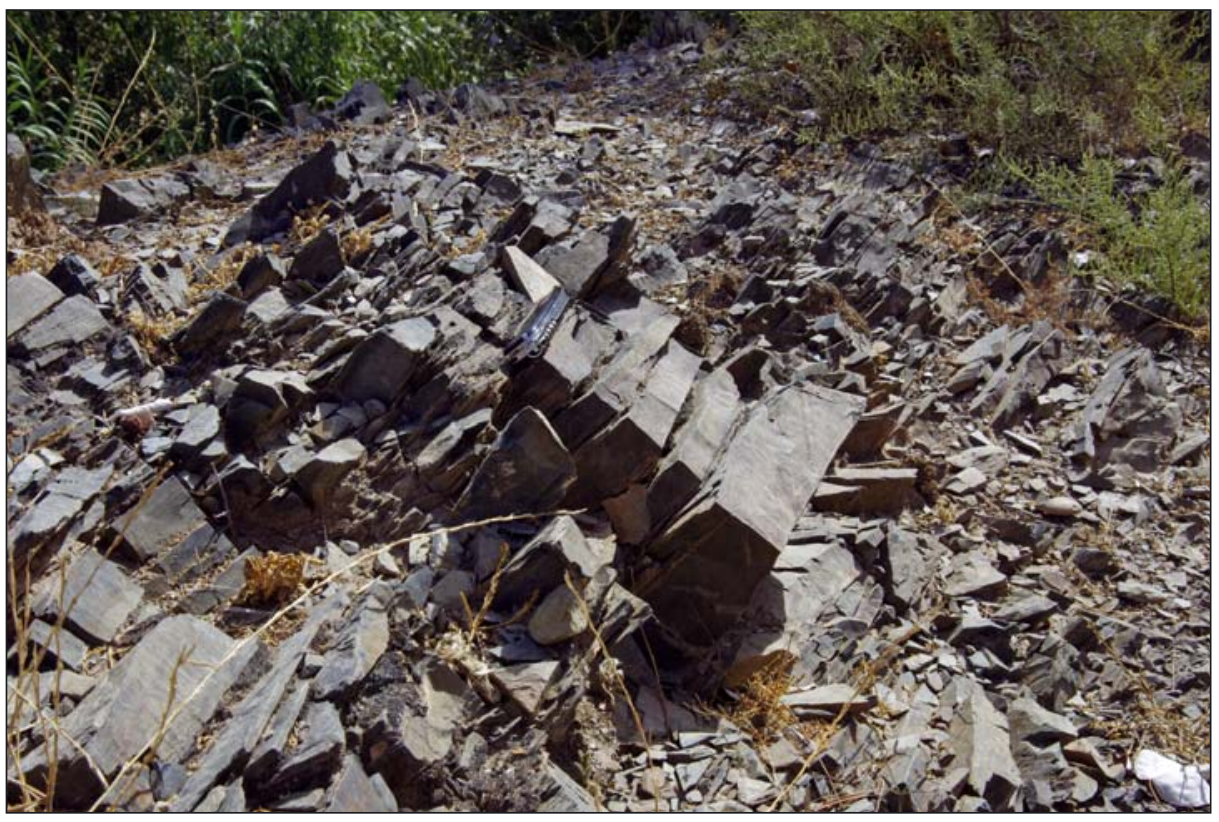

Abb. 4: Tonschiefer am westlichen Ufer des Nfys bei der Oase Nfys 
Bei der kleinen Oase Nyfs hat sich der gleichnamige Fluss tief in den anstehenden Schiefer eingegraben. Etwas oberhalb der Oase wird der Fluss heute durch eine Staumauer zu einem ausgedehnten See aufgestaut. Unser weiterer Weg verlief deshalb auf Umwegen um den ausgedehnten Stausee herum.

\section{\#7 Amismiz}

„Als wir abends gegen 6 Uhr die Thore der Qasbah überschritten hatten, wies man uns einen freien, von Mauern und Gärten umgebenen Platz an, wo wir unsere Zelte aufschlagen konnten." (LeNz 1884, Bd. 1, S. 266)

Die moderne Stadt am Fuß des Hohen Atlas liegt am östlichen Flussufer auf einem Hügel. Sie ist umgeben von gepflegten Gärten und Olivenhainen. Es sind ausnahmslos nur noch moderne Gebäude vorhanden. Von der alten Kasbah ist nichts mehr zu sehen.

In Amismiz erlebte LENZ eine einschneidende Änderung seines Reiseweges. Vom direkten Weg nach Süden über den Hohen Atlas wurde ihm hier wegen des zu beschwerlichen Abstiegs vom Pass abgeraten. Somit musste er einen bedeutenden Umweg nach Westen und Nordwesten gehen, um den einfacheren Pass von Bibauen zu erreichen.

\section{\#8 westwärts}

„Am 8. März, morgens 8 Uhr, brachen wir auf und wandten uns zunächst westwärts. Das Plateau war hier von zahlreichen Kanälen durchzogen, die zur Bewässerung der Gerstenfelder dienen ..." (LENZ 1884, Bd. 1, S. 267)

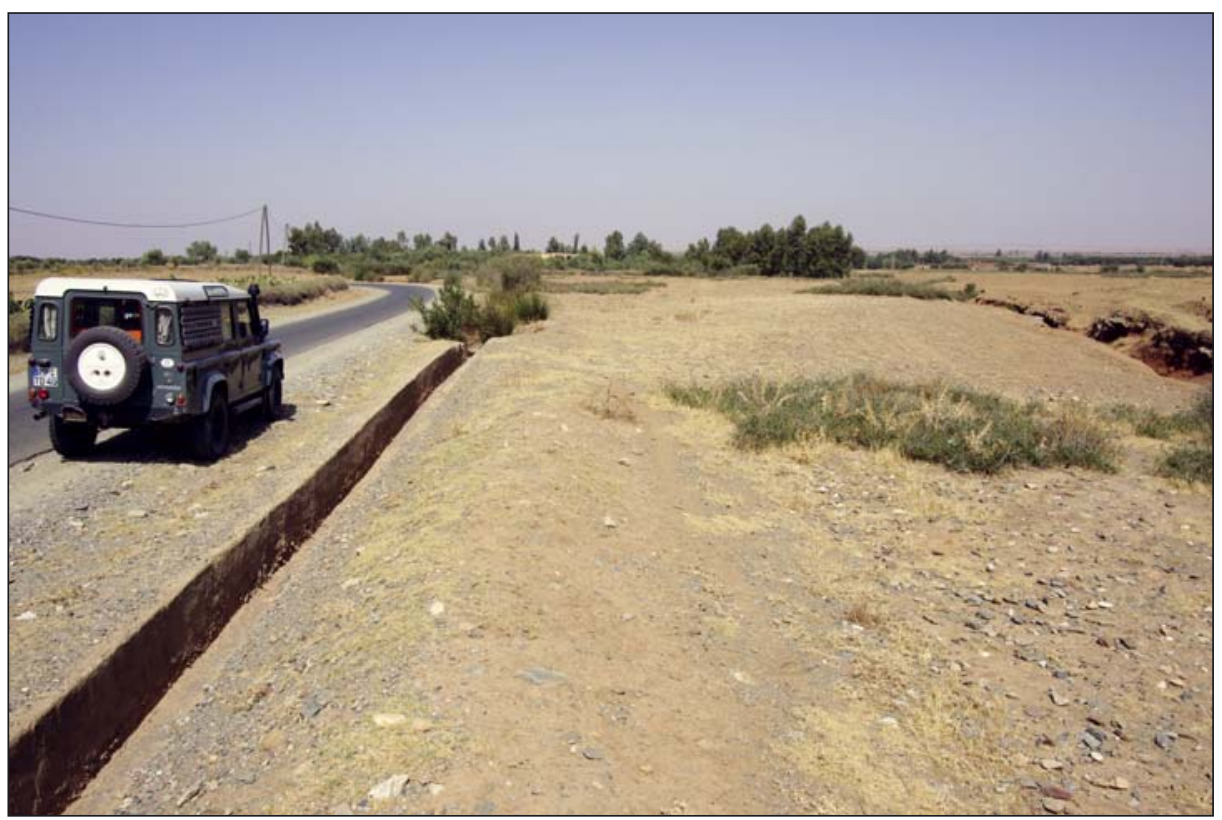

Abb. 5: westwärts, Kanäle 
Auf einer kaum befahrenen schmalen Straße fuhren wir parallel zum Hohen Atlas nach Westen. Abgehend von den natürlichen Entwässerungsrinnen, vom Hohen Atlas ausgehend, verlaufen künstliche, befestigte und unbefestigte Kanäle in Ost-WestRichtung.

\section{\#11 Wadi el Mel}

„Dann kamen wir auf ein sehr steiniges unfruchtbares Plateau, welches von einem tiefen Wad mit sehr steilen Wänden, dem Wad-e1-Mel (oder W. Asif-el-Mel) durchzogen wird, der sich später mit dem Tensift vereinigt. "(LENZ 1884, Bd. 1, S. 267)

Der Fluss kommt aus einem breiten, gut sichtbaren Tal des Hohen Atlas und fließt nach Norden. Das gegenüberliegende Ufer steigt fast senkrecht mehrere Meter hoch an. So wie LeNZ mussten auch wir nach Norden ausweichen. An den Flussrändern gibt es ausgedehnte landwirtschaftlich genutzte Flächen und Olivenhaine.

\section{\#12 nordwärts}

„Wir zogen eine Strecke nordwärts parallel dem Wad-el-Mel.“ (LENZ 1884, Bd. 1, S. 267)

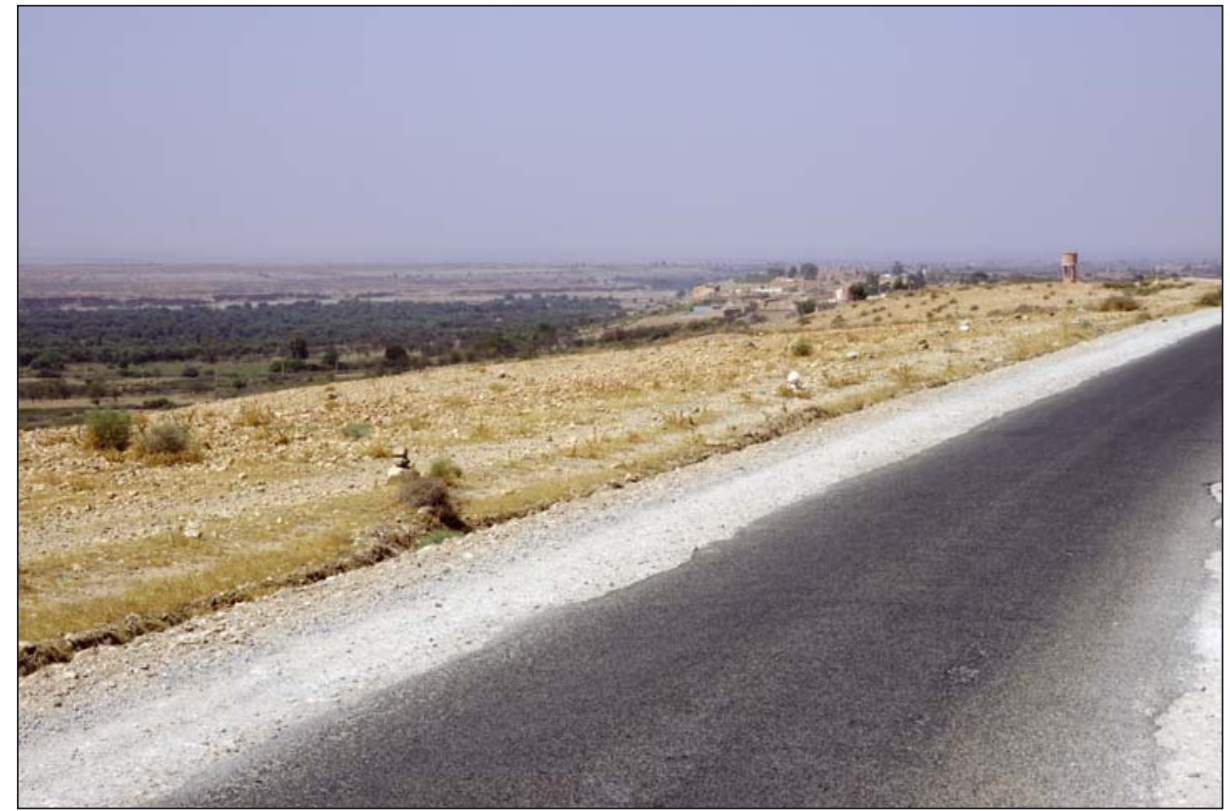

Abb. 6: nordwärts am Wadi El Mel entlang

Unsere bisherige westliche Reiserichtung wurde durch den Fluss nach Norden gelenkt. Erst nach etwa zehn Kilometern quert die schmale Straße den Fluss auf einer kleinen Brücke. Der Fluss war ganz trocken. 


\section{\#13 Darakimacht}

„... und hielten gegen 3 Uhr am rechten Ufer des Flusses in einem kleinen Dorfe, Darakimacht, das von den Berbern der Kabyle Amsmiz bewohnt wird. Die infolge des steinigen schlechten Weges stark ermüdeten Thiere konnten nicht mehr weiter, und wir mussten in dem kleinen Flecken die Nacht in ziemlich dürftiger Weise verbringen. “ (LENZ 1884, Bd. 1, S. 267-268)

Der genannte kleine Ort ist heute unter dem Namen Dar-Akimakh in den Landkarten eingetragen und konnte daher eindeutig zugeordnet werden. Der Ort selbst besteht aus wenigen Gehöften und niedrigen Lehmhäusern.

\section{\#14 Die Kasba Mzudi}

„Am folgenden Morgen brachen wir zeitiger auf. [...] Es war ziemlich schwierig, die schwer bepackten Tragthiere den steilen Abhang hinab und am jenseitigen Ufer wieder hinaufzubringen, aber es gelang ohne weitere Unfälle und schon gegen $11 \mathrm{Uhr}$ langten wir vor der Qasbah des Kaid von Mzudi an. Vor derselben, die mit einer hohen starken Lehmmauer umgeben war, durch welche ein schmales Thor führt, befindet sich ein hübscher freier Platz mit etwas Buschwerk, und wir erhielten die Erlaubniss, hier unsere Zelte aufschlagen zu dürfen." (LENZ 1884, Bd. 1, S. 268)

Das Auffinden dieser Örtlichkeit gestaltete sich sehr schwierig. Die Lokalität einer Kasba mit dem Namen Msoudi wurde lediglich auf einer alten Karte (MAroc 1949, Nr. LII) gefunden. Die Koordinaten des Punktes wurden aus der in GON skalierten und auf den Nullmeridian in Paris kalibrierten Karte mit N34 ${ }^{\mathrm{G}} 71,05$ W12 13 , 77 ermittelt. Nach der Umrechnung in die Winkelmaßeinheit Grad und den Nullmeridian von Greenwich konnte auf einem aktuellen Satellitenbild unweit dieser Stelle ein großes Bauwerk entdeckt werden.

Zur Vergewisserung, ob es sich bei diesem ermittelten Gebäude um die gesuchte Kasba Mzudi handelt, wurde noch folgende Überprüfung mit verfügbaren Daten vorgenommen:

- Die Peilung vom letzten bekannten Punkt \#13 Darakimacht auf den oben genannten ermittelten Punkt ergibt eine Luftlinien-Entfernung von ca. 13,5 Kilometern in der von LENZ beschriebenen westsüdwestlichen Richtung. Seine tatsächlich zurückgelegte Strecke war natürlich größer, da ein Marsch auf der kürzesten Verbindungsstrecke, bedingt durch das Gelände, nicht immer möglich war.

- Lenz war an diesem Tag etwa vier Stunden unterwegs (vom zeitigen Aufbruch gegen 7 Uhr bis $11 \mathrm{Uhr}$ ). Eine beladene Kamelkarawane kann etwa 3,5 bis $4 \mathrm{~km} / \mathrm{h}$ zurücklegen. LENZ konnte also in seinen vier Stunden Marsch eine Strecke von 14 bis 16 Kilometern bewältigen. Dies deckt sich ausreichend genau mit der ermittelten Luftlinien-Entfernung.

Da also sowohl der in der Karte gefundene Ort, die Richtung und die Entfernung zu dem bekannten vorherigen Ort mit den Angaben von LeNZ übereinstimmten, konnten wir davon ausgehen, dass der besuchte Ort die von Lenz beschriebene Kasba Mzudi ist. 
Als wir den berechneten Ort erreichten, fanden wir einen weitläufigen Baukomplex mit hohen Mauern aus traditionellem Stampflehm und Natursteinen vor. Die äußeren Umgebungsmauern und kleinere Gebäude im Innern der Anlage sind teilweise eingefallen. Im Zentrum der Kasba befinden sind noch intakte, zweigeschossige Gebäude. Einige Räume davon sind noch bewohnt und werden gepflegt. Die Bewohner konnten leider keine Auskunft über die Historie und den Namen des Bauwerks geben. An der Südseite der ungefähr acht Meter hohen Umfassungsmauer befindet sich ein Eingangstor mit Resten von Verzierungen.

\section{\#18 Imi N Tanout}

„Bei dem nur eine starke Stunde von Seksaua gelegenen Orte Imintjanut betraten wir das eigentliche Atlasgebirge. Der Ort ist wichtig, da von hier die meisten Karavanen, die zwischen Marrakesch und Wad Sus verkehren, den Uebergang über die Berge bewerkstelligen." (Lenz 1884, Bd. 1, S. 271)

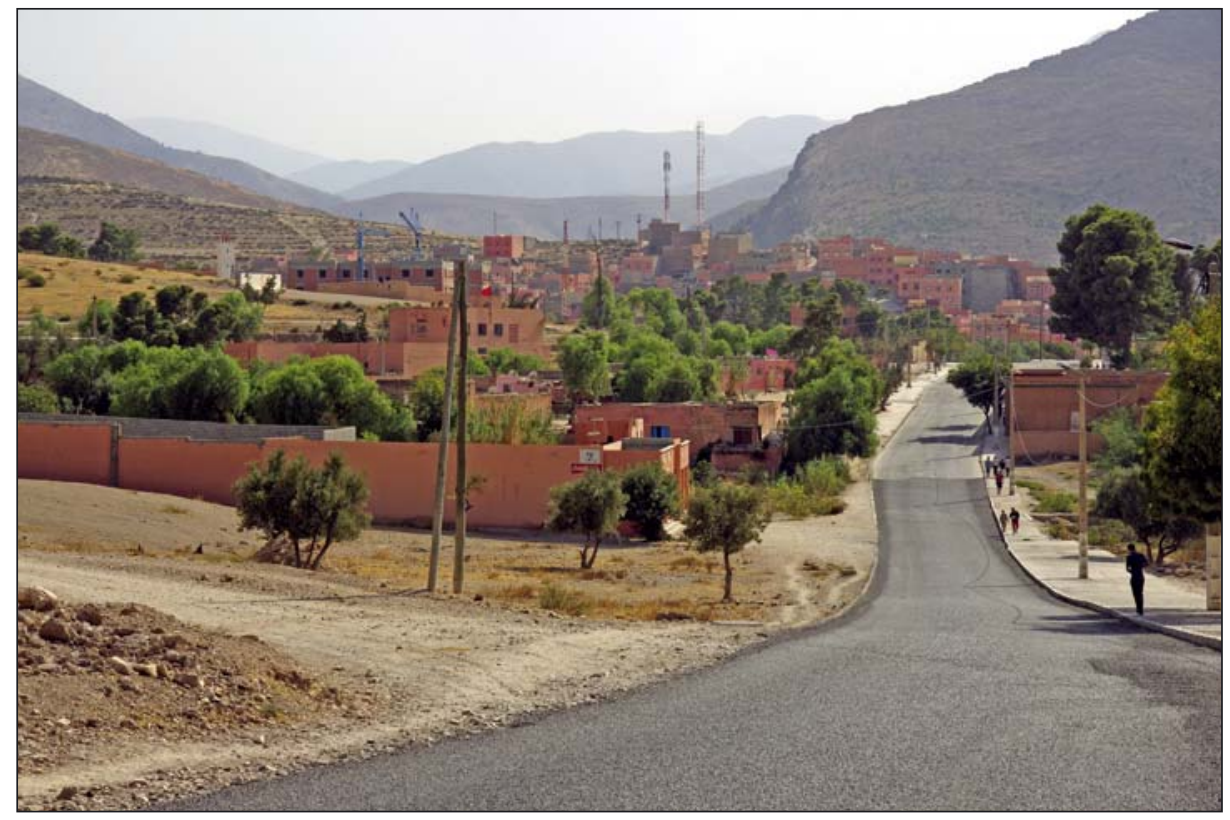

Abb. 7: Imi N Tanout; Blick nach Süden in den Hohen Atlas

Von einer höher gelegenen Ebene kommend sahen wir den modernen Ort vor uns liegen, und direkt dahinter erhoben sich ganz nah die Berge des Hohen Atlas. Das Tal in südlicher Richtung, dem wir dann folgten, war deutlich zu erkennen. Es war der Zugang in den Hohen Atlas, dem wir jetzt seit Amizmiz parallel nach Westen gefolgt sind, und der Beginn der Überquerung dieses Gebirges. 


\section{\#19 nach Süden}

„Wir ritten erst eine Stunde lang in rein südlicher Richtung; zu beiden Seiten hatten wir die steil aufgerichteten Schichten lichter Kalke und Kalkmergel, ..." (LENZ 1884, Bd. 1, S. 272)

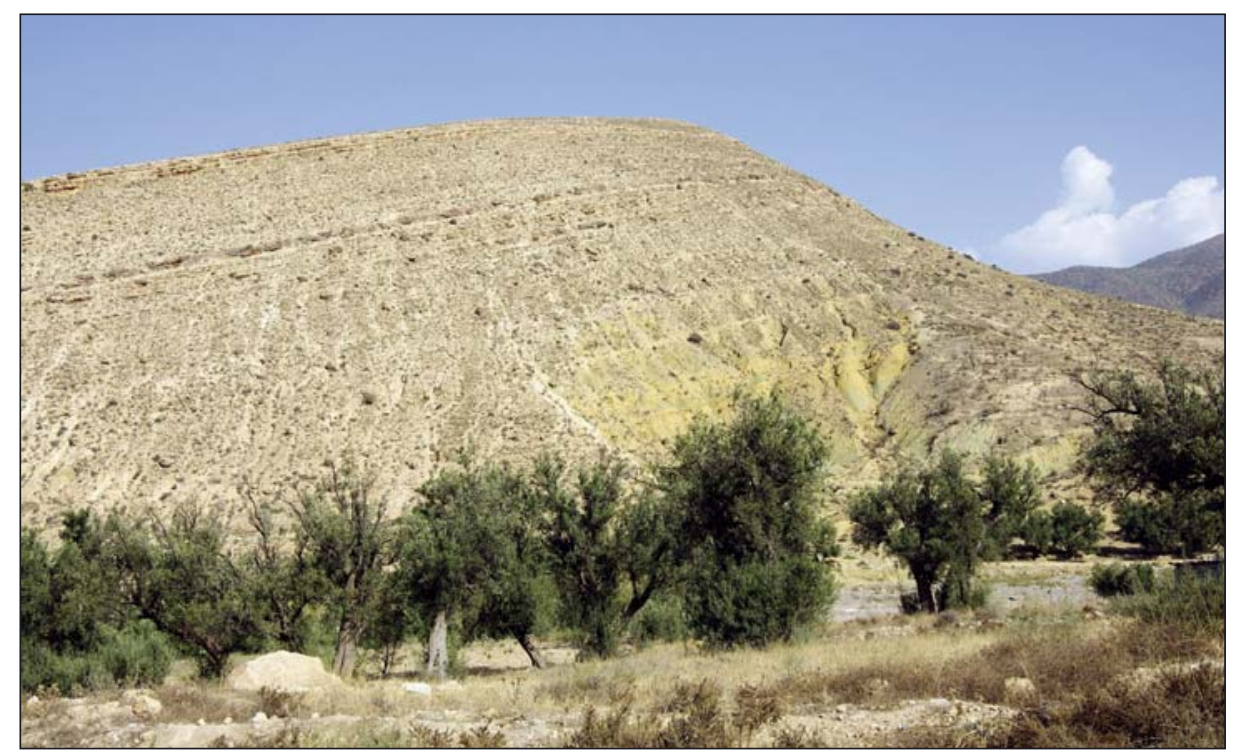

Abb. 8: nach Süden; Gesteinsstufen mit Kalkmergel

Durch ein schmales Tal führt ein gute Straße nach Süden in die Berge und verläuft am rechten Talhang entlang; am linken Talrand war ein schmaler unbefestigter, von Eseln benutzter Pfad zu erkennen. Deutlich waren die Gesteinsschichten in den aufgefalteten Bergen zu sehen. Im unteren Bereich trat der weiche, grüne Kalkmergel zu Tage.

\section{\#20 nach Westen}

„Der schmale Weg führte längs des linken Thalgehänges und bog dann plötzlich unter rechtem Winkel in ein breites schönes Längsthal ein, dem wir westwärts mehrere Stunden folgten. Wir begegneten nur äusserst selten einem Menschen, aber das Thal ist gut bebaut; neben Feldern von Getreide fiel mir hier vor allem die Pflege der Mandelbäume auf, die, in Blüte stehend, in grossen Mengen sich finden und treffliche Früchte geben." (LENZ 1884, Bd. 1, S. 272)

Nach etwa 3,5 Kilometern seit Imi N Tanout biegt die Straße nach Westen ab. Dies entspricht auch der von LENZ genannten Reisezeit von einer Stunde. Auf der weiteren Fahrt nach Westen begleiteten uns beiderseits der Straße bewirtschaftete Felder und niedrige Mandelbäume. 


\section{\#26 roter Sandstein}

„Die Berge bestehen noch immer aus dem rothen Sandstein, der völlig versteinerungslos zu sein scheint." (LENz 1884, Bd. 1, S. 276)

Das Erscheinungsbild der Landschaft änderte sich merklich, da ringsum nur noch intensiv roter Sandstein vorherrscht.

\section{\#29 Bach Ait Moussa}

„Wir passirten den District Ait Musa mit einem grossen Freitags-Soko (Wochenmarkt); auch der im Thale fliessende nicht unbedeutende Bach führt diesen Namen." (LENZ 1884, Bd. 1, S. 277)

Etwas südlich der Ortschaft Argane querten wir den Bach Ait Moussa über eine Betonfurt.

\section{\#31 Pass Bibauan}

„Wir hielten abends auf der Wasserscheide des Atlasgebirges, etwas über $1200 \mathrm{~m}$ hoch, in einer zur Zeit völlig unbewohnten Gegend. Reste der zerstörten Dörfer sah man allenthalben. Die Gegend war wunderschön." (LENz 1884, Bd. 1, S. 277)

Vom Tal des Moussa aus schlängelt sich ein schmaler, einspuriger Asphaltweg nach Südsüdost in die Berge hoch. Über unzählige Serpentinen und über bewaldete Berghänge geht es vorbei an kleinen belebten Ortschaften. Die roten Häuser haben flache, mit Erde bedeckte Dächer. Vom Pass aus hat man einen weiten Blick nach Süden. Etwas unterhalb des Passes konnten wir auf einem kleinen, ebenen Stellplatz unser Nachtlager aufschlagen und in Ruhe des Durchzugs von Oskar LENZ an dieser Stelle am 13. März 1880 gedenken. Auf der Ostseite der Berghänge sieht man vom Pass aus abseits des Asphaltwegs noch mehrere schmale, alte ausgetretene Pfade, die nach Süden ins Tal führen.

Der Name des Passes Bibauan existiert nur in der Angabe von Lenz. Er wird hier übernommen, aber er wurde nicht in den verwendeten Karten gefunden.

\section{\#34 El-Menizla}

„... und gegen Abend langten wir ungefährdet in der Stadt Emnislah an, deren Häuser wir schon lange vor uns gesehen hatten. Ein Blick rückwärts zeigte uns erst, was für einen schwierigen Weg wir zurückgelegt hatten. [...] Emnislah, ein Doppelstädtchen, da an beiden Seiten des Thales eine Häuserpartie steht, liegt ebenso am Südabhang des Atlas wie Imiutjanut am Nordabhang und hat die selbe Bedeutung für Karavanen, welche von Sus aus nach Marrakesch mit Benutzung des Bibauan-Passes reisen wollen." (LENZ 1884, Bd. 1, S. 279) 


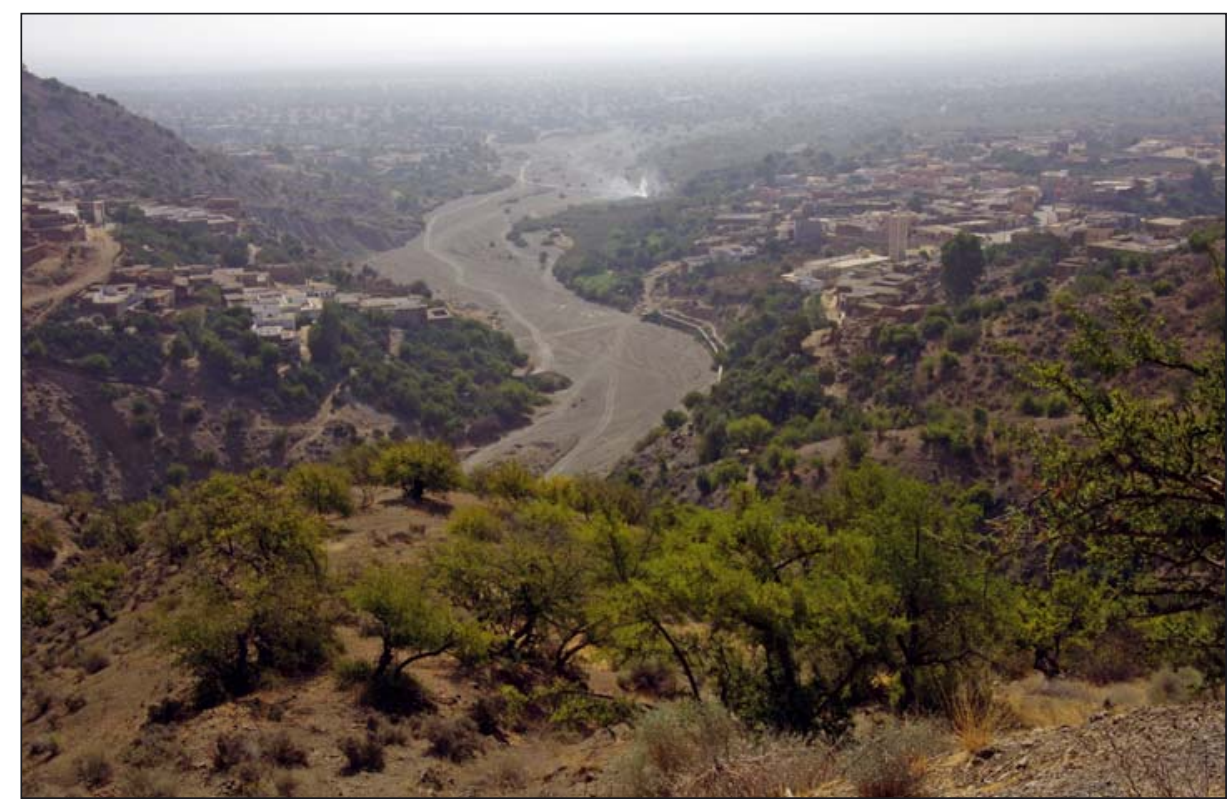

Abb. 9: El-Mienizla von Norden

Auf weiterhin gutem Asphaltweg mit engen Kurven, vorbei an vereinzelten, bewohnten Gehöften, ging die Abfahrt vom Pass an die Südseite des Hohen Atlas gut voran. Immer wieder boten sich Ausblicke auf das flache Land jenseits der Berge mit dem fruchtbaren Wadi Sus. Kurz vor dem Ende der Abfahrt ist die Stadt El-Menizla am Fuß der Berge zu sehen. Sie liegt auf beiden Seiten eines trockenen Flusstales. LENZ zählte die weitere Strecke bis Taroudant zu den gefährlichsten Partien in Nordafrika - aufgrund von Raubzügen durch den Stamm der Howara. Deshalb schloss er sich zum Schutz mit einer größeren Handelskarawane zusammen und ritt kampfbereit mit dem Revolver in der Hand weiter.

\section{\#37 Gaba}

„Etwas ausserhalb der Stadt hatten wir die Ruinen der angeblich römischen Stadt Gaba zur Seite liegen lassen." (Lenz 1884, Bd. 1, S. 283)

Auf der Karte „Taroudant“ (Maroc 1946, Nr. LXX) ist eine Ruine mit dem Namen Gaba bei $\mathrm{N} 33^{\mathrm{G}} 90 \mathrm{~W} 12^{\mathrm{G}} 55,05$ eingetragen. Diesen Punkt haben wir angefahren, da bei der Vorbereitung der Route auf einer Satellitenkarte kleine Häusergruppen und ein Brachgelände mit Bodenstrukturen zu sehen waren, die eventuell alte Grundmauern sein konnten. An Ort und Stelle wurde zwar der Name der Siedlung mit Gaba bestätigt, aber mehrere Auskunftspersonen wussten nichts von alten römischen Ruinen. Da das Gelände bis vor die Tore von Taroudant fast vollständig mit eingezäunten Plantagen bedeckt ist, war dort eine weitere Suche nach Ruinen nicht möglich. 


\section{\#38 Taroudant}

„Es war noch zeitig am Tage, erst gegen 2 Uhr, als ich Tarudant am 15. März 1880 erreichte, und hatte ich damit wieder ein Stück meines Vorhabens, und nicht das geringste, zurückgelegt“ (LENZ 1884, Bd. 1, S. 283)

„... Wir passierten das nördliche Thor und zogen, möglichst das Innere der Stadt vermeidend, längs der Stadtmauer der im Nordosten der Stadt gelegenen Qasbah zu. “ (LENZ 1884, Bd. 1, S. 292)

Durch ein seit Gaba andauerndes Gewirr von engen, unübersichtlichen Wegen zwischen eingezäunten Plantagenpflanzungen erreichten wir die erste Hauptverkehrsstraße nach der Querung des Hohen Atlas. Diese führte uns direkt auf das westliche Tor der vollkommen intakten, die gesamte Altstadt umgebenden Stadtmauer von Taroudant zu.

Wir besuchten auch das nördliche Tor, das Bab Ouladbonona, durch das LENZ in die Stadt einzog. Es ist vollkommen erhalten.

\section{\#40 Wadi Sus}

„Der Weg führte etwas südlich, wir überschritten den kleinen Wad Dschitarin kurz oberhalb seiner Vereinigung mit dem Wad Sus und gelangten bald an diesen selbst. Das eigentliche Flussthal ist sehr breit, aber wenig tief, da die Steilränder nur geringe Höhe haben; der grösste Theil des Bettes ist vollständig mit feinem Sande angefüllt und der Fluss selbst bestand zu meiner Zeit aus einer kaum 10-12 Fuss breiten und 1-2 Fuss tiefen Wasserrinne. Ich hatte einen gewaltigen Strom erwartet und fand nur diesen schmalen Streifen fliessenden Wassers. [...] Die Ueberschreitung des Flusses machte demnach nicht die geringste Schwierigkeit." (LENz 1884, Bd. 1, S. 316-317)

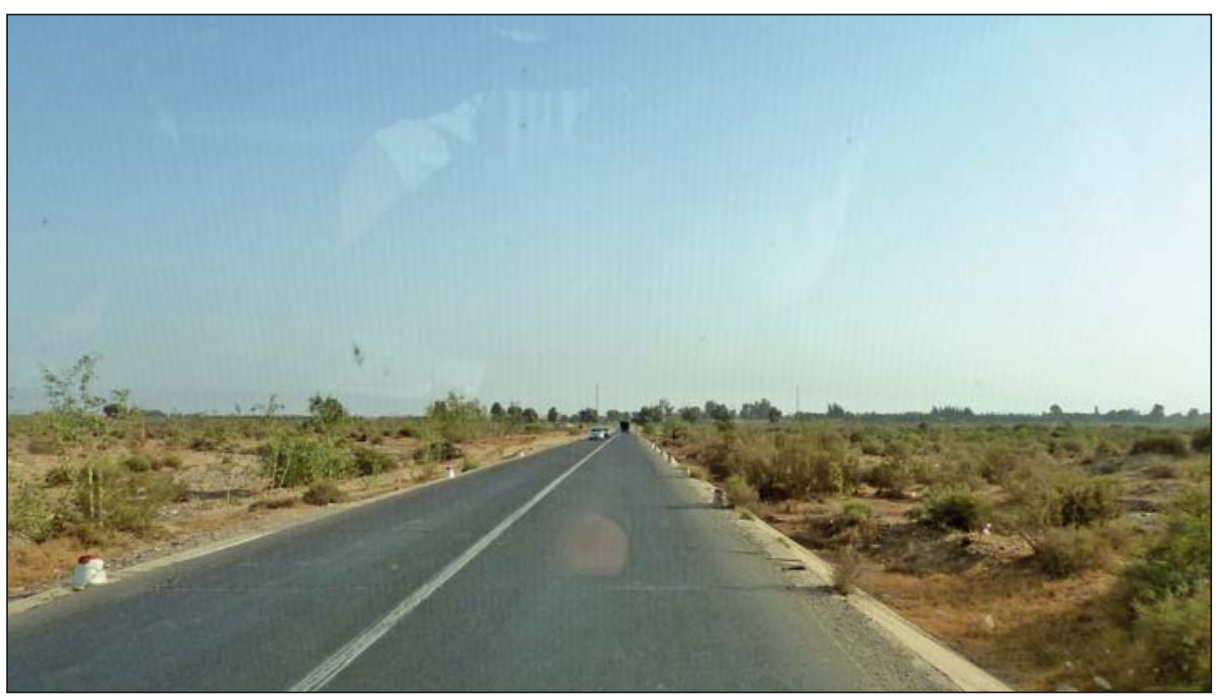

Abb. 10: Straße durch das Wadi Sus 
Wir überquerten das trockene Flusstal auf einer guten Teerstraße. Das Flussbett ist an dieser Stelle über einen Kilometer breit und mit niedrigen Büschen bewachsen. Kurz vor dem flachen, südlichen Ufer führt eine kleine Brücke über einen schmalen Streifen langsam fließenden Wassers.

\section{\#43 Ida Mennou}

„Wir ritten erst in westlicher Richtung bis zur Ortschaft Ida Menou meist durch bebaute und eingezäunte Felder, auch einzelne Partien von Arganwäldern ...; wir passirten hierauf eine aus Kalksteinen bestehende Hügelkette ..." (LENZ 1884, Bd. 1, S. 318)

Wir fuhren durch eine ausgedehnte Kulturlandschaft abgeernteter Äcker mit vielen kleinen Ortschaften weiter nach Süden. Im Osten liegen die Ausläufer des Antiatlas. In Ost-West-Richtung verläuft eine Hügelkette.

\section{\#49 Sidi Said ben Meza}

„Die Richtung, die wir einschlugen, war im allgemeinen eine südwestliche. Wir passirten eine Reihe von gut bevölkerten Gegenden mit zahlreichen Ortschaften, wie Ait Wadrim, Ait Midik, mit der Zauja Sidi Said ben Meza ..." (Lenz 1884, Bd. 1, S. 319)

Diese Zauja (oder Zawija) ist eine weiß gekalkte Grabstätte für einen islamischen Heiligen, die man in Marokko Marabout nennt. Das Gebäude ist gepflegt und vollständig intakt. Es steht inmitten eines etwa 200 mal 300 Meter großen Areals. Es ist dicht bewachsen mit Wüstenheide, die rosa blüht. Auch zahlreiche alte Gräber befinden sich hier. Die Lokalität dieser Zawija konnte auf der Karte (Maroc 1949, Nr. LII) gefunden und bestimmt werden. Wenige hundert Meter südlich des Gebäudes beginnt die Wasserfläche des Stausees Youssef ben Tachfine. Dieser hinderte uns daran, ab dieser Stelle weiterhin in eine südliche Richtung zu fahren.

\section{\#55 Ilerh}

„Am 30. März führte uns wieder ein langer Marsch in die Residenz des kleinen selbständigen Staates, der auf den Karten gewöhnlich als das Land des Sidi Hescham bezeichnet wird. Bei strömendem Regen, stark durchnässt und schon bei völliger Dunkelheit trafen wir in dem Städtchen Ilerh ein." (LENz 1884, Bd. 1, S. 321)

Wir erreichten Ilerh (heute Illirh) von Osten kommend auf einer steinigen Piste. Sofort fielen uns die bis zu acht Meter hohen Mauerreste und Ruinen auf, die aus traditionellem Stampflehm erbaut wurden. Der kleine bewohnte Ort liegt südlich von diesem Ruinenfeld. Neben kleinen modernen Gebäuden werden auch noch traditionelle alte Kasbahs mit gepflegten Innenhöfen und bemalten Fassaden bewohnt. 


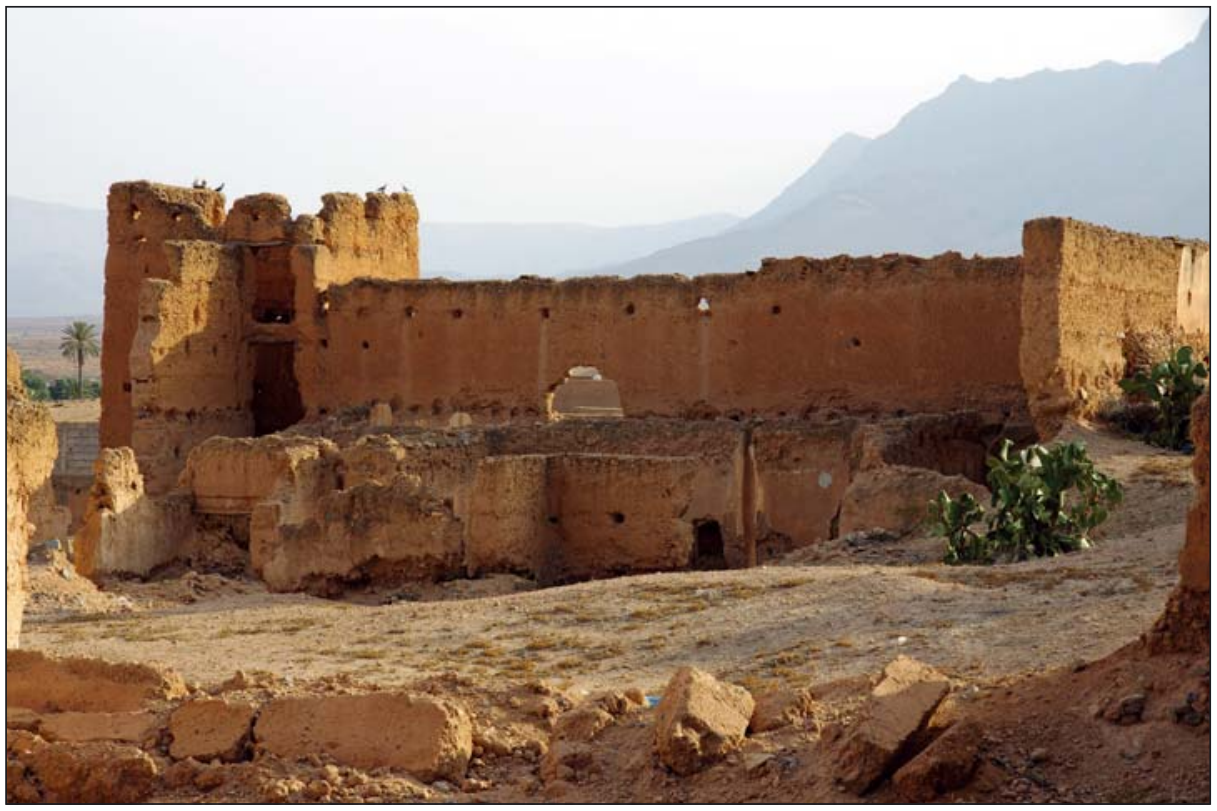

Abb. 11: Ruine in Ilerh

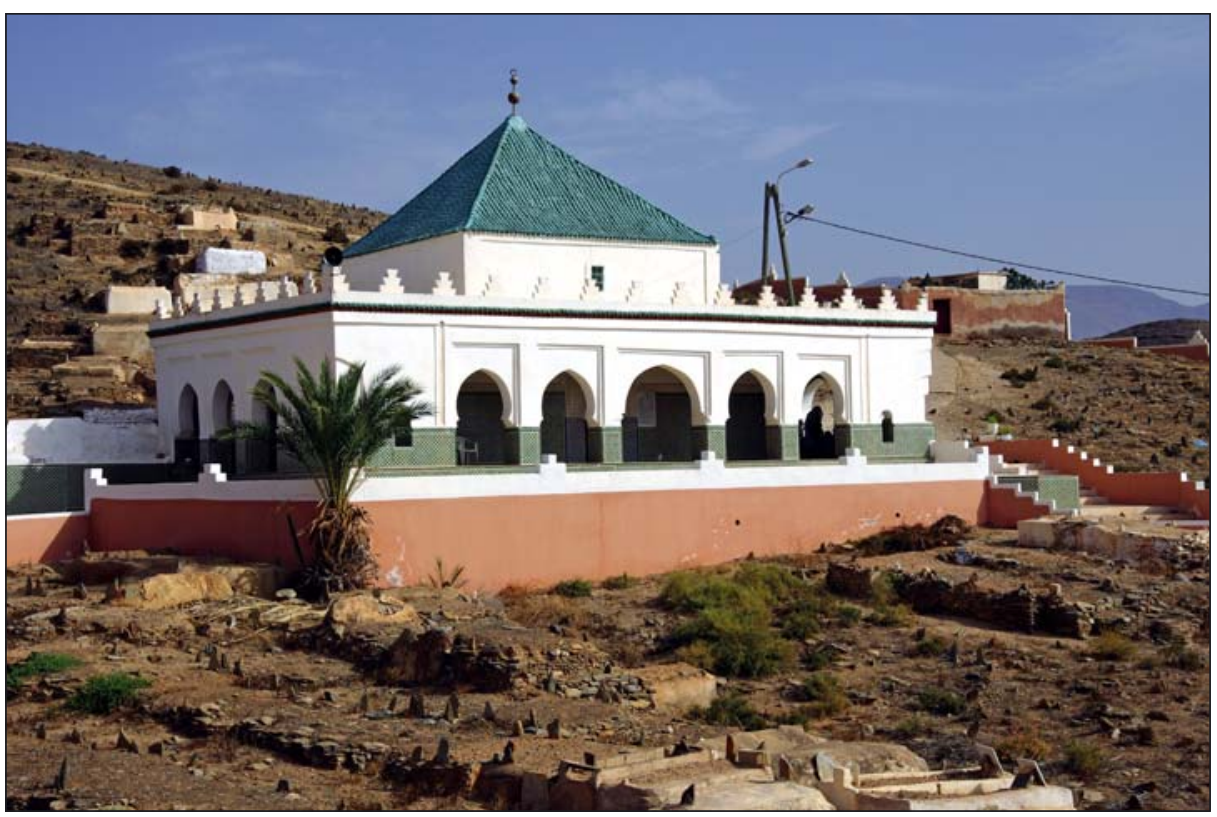

Abb. 12: Zaoui-Sidi Ahmed ou Moussa 


\section{\#56 Zauja Sidi Hamed ben Musa}

„Eine Stunde von der Stadt entfernt wird bei der Zauja Sidi Hamed ben Musa dreimal jährlich ein grosser Markt abgehalten, zu welchem, wie erwähnt, selbst aus weiter Ferne Händler herbeikommen. Aus Marrakesch sogar kommen die Kaufleute und scheuen den Weg über den Atlas und durch die dann folgenden unsichern Howaragebiete nicht, um hier Geschäfte zu machen." (LENZ 1884, Bd. 1, S. 323-324)

Etwa sechs Kilometer östlich von Illirh liegt der heutige Ort Zaoui-Sidi Ahmed ou Moussa. Lenz hat diesen Ort zwar selbst nicht besucht, sich aber von dort mit Waren für seine weitere Reise versorgen lassen. Heute ist der Ort mit der gleichnamigen großen und sehr gepflegten Grabstätte ein bedeutender Wallfahrtsort.

\section{\# 58 Fuß einer Bergkette}

„Wir folgten nun anfangs dem Thal des Wad Tazzerult aufwärts und gelangten bald an den Fuss einer Gebirgskette, die in der Richtung von Südwest nach Nordost streicht, also dem Atlassystem angehört und vorherrschend aus Granit und Schiefer besteht. Der nach Norden zu gelegene Abhang ist ein sehr steiler Abbruch." (LENZ 1884 , Bd. 2, S. 3)

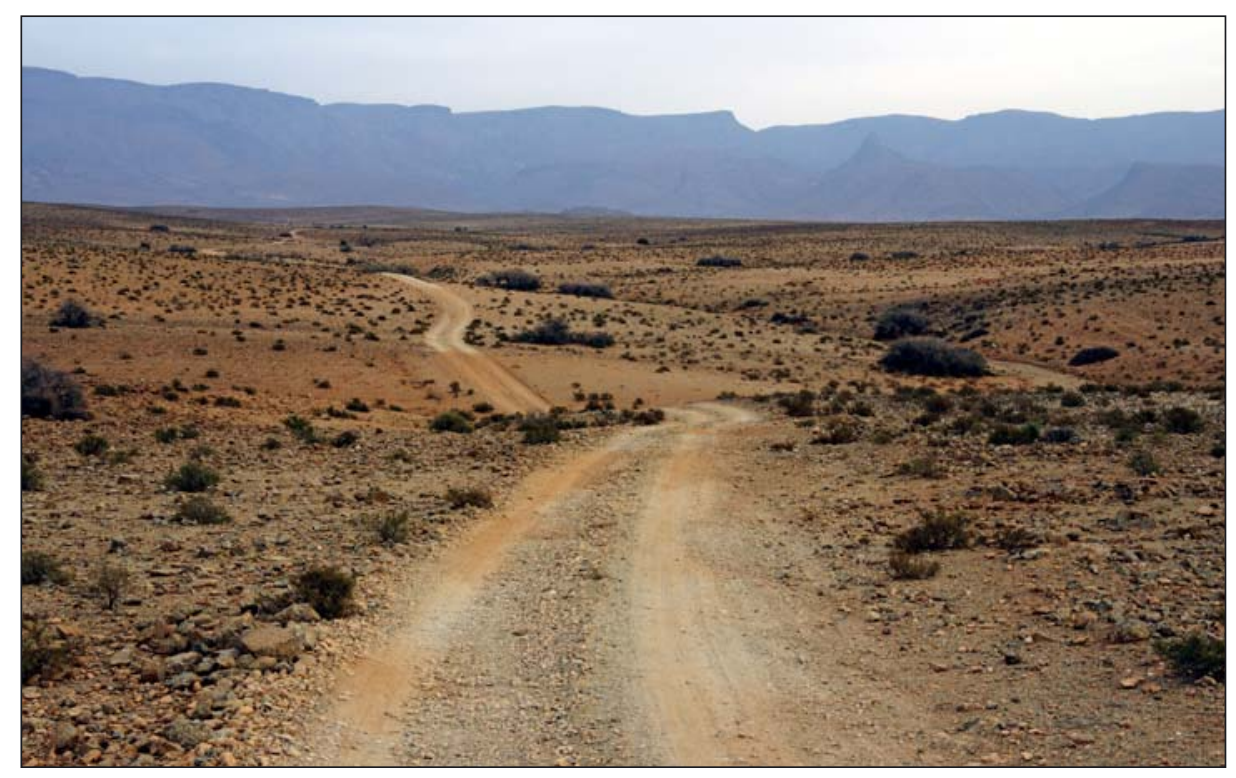

Abb. 13: Fuß einer Bergkette

Über eine große Hochebene fuhren wir auf einer kleinen Piste östlich des ausgetrockneten Wadi Tazzerlut weiter nach Süden. Die steil aufragende Bergkette lag weit sichtbar vor uns. Die von LENZ angegebene Richtung Südwest/Nordwest trifft zu. 


\section{\# 59 Pittoresker isolierter Kegel}

„Auch Eruptivgesteine treten hier auf und bilden sehr pittoreske isolirte Kegel, auf deren einem Sidi Hussein eine Citadelle besitzt. Der Anblick dieser Agadir genannten kleinen Festung auf der höchsten Spitze eines steil aufragenden, völlig unzugänglich erscheinenden Felsenkegels ist im höchsten Grade imposant. “(LENz 1884, Bd. 2, S. 3)

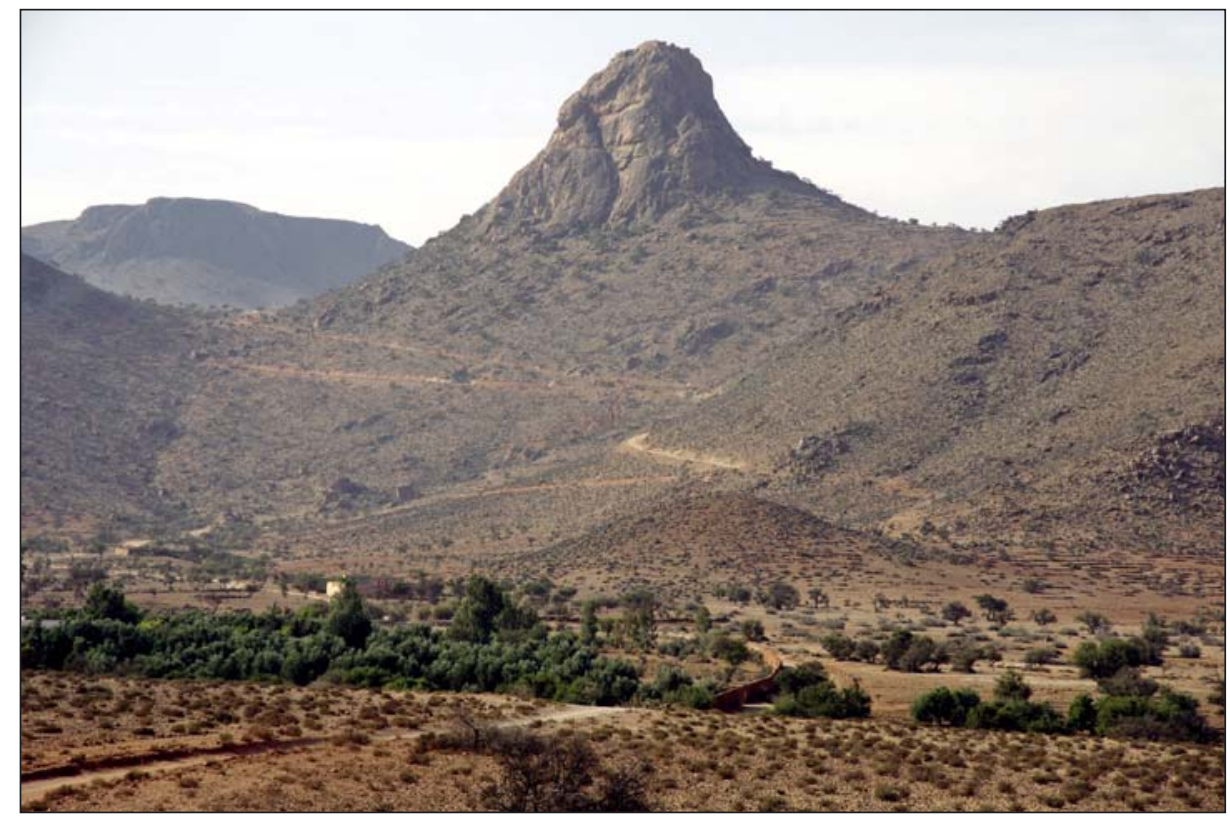

Abb. 14: pittoresker isolierter Kegel

Wir waren natürlich sehr gespannt diese doch sehr interessant beschriebene Landmarke zu finden. Und schon in Illigh waren wir erfreut, als wir vom Dach einer Kasbah aus diesen imposanten Kegelberg in der Ferne sahen. Eine unbefestigte steinige Piste führte uns zum Teil über Serpentinen direkt auf den Berg zu. Auf der Bergspitze sind Reste von Mauern der Speicherburg zu erkennen. Am Fuß der Bergspitze waren einige Zisternen vorhanden. Einen direkten Zugangsweg auf die Bergspitze über den glatten Fels gibt es nicht mehr. Reste dieses gemauerten Weges kleben noch am Fels.

\section{\# 61 östlich}

„Nach einem mehrstündigen Marsch auf zwar steilen, aber relativ guten, in zahllosen Serpentinen ansteigenden Wegen erreichten wir den Kamm des Gebirges. [...] Wir wandten uns nun östlich." (Lenz 1884, Bd. 2, S. 4) 


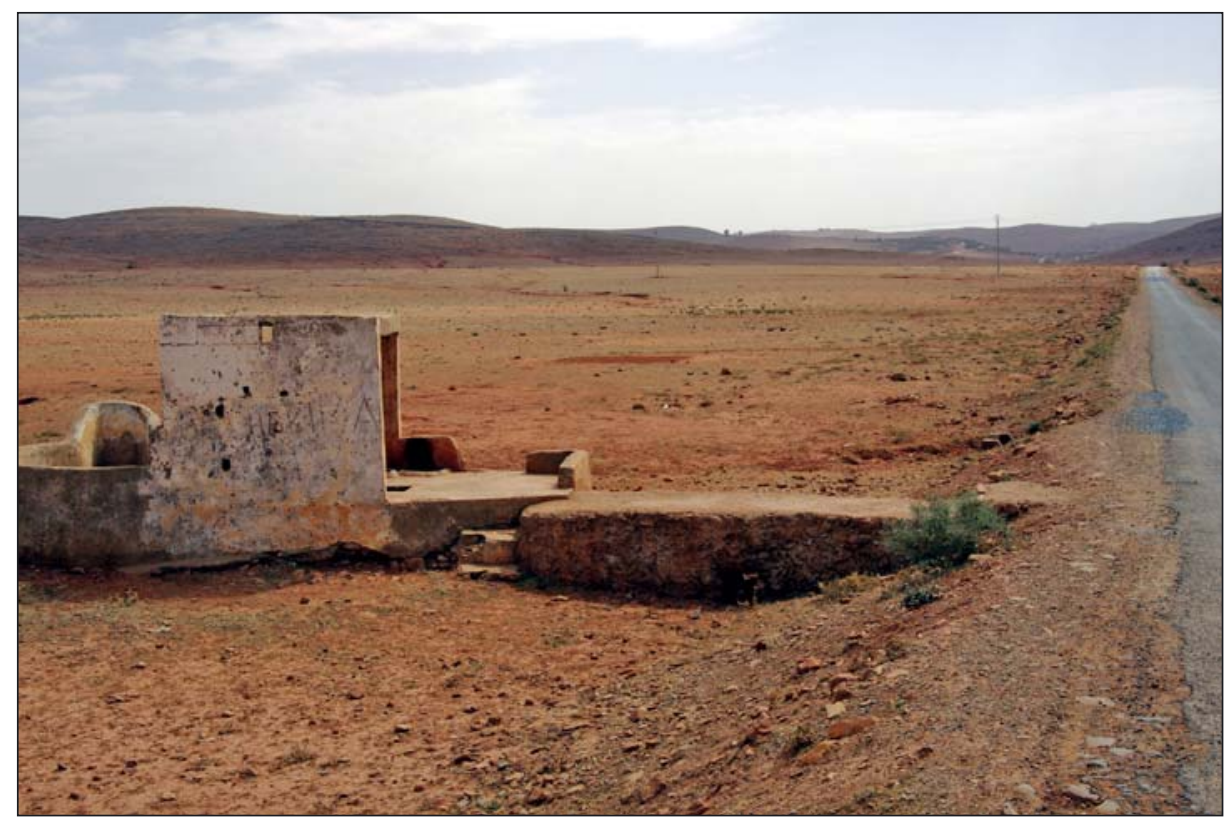

Abb. 15: nach Osten

Auf zahlreichen Serpentinen einer einspurigen unbefestigten Erdpiste erklommen wir ebenfalls den Bergkamm. Dieser Kamm ist eigentlich der Rand der nach Osten hin sich ausdehnenden gewellten Ebene. Von Osten her betrachtet ist der Kamm bei weitem nicht so ausgeprägt wie vom Norden.

Ob wir den Spuren von LENZ in südöstlicher Richtung durch das offensichtlich freie und dünn besiedelte Gelände folgen könnten, war schon in der Reisevorbereitung zweifelhaft. Und an Ort und Stelle bestätigte sich, dass das grobsteinige Gelände eine Fahrt ohne Wege so gut wie nicht zulässt. Deshalb benutzten wir einen etwas nördlich gelegenen Weg, um anfänglich zumindest in Ostrichtung und dann in Südostrichtung fahren zu können. Das Gelände war zunächst stark gewellt mit schräg stehenden Felsschichten und später von Flusstälern tief zerschnitten.

\section{\# 65 Wadi Temenet}

„Der Weg führte in Zickzacklinien durch ein gebirgiges, unbewohntes, ödes, wasserarmes Gebiet. [...] Gegen 11 Uhr betraten wir das breite, wasserlose Thal des Wad Temenet, der einige Stunden unterhalb Fum-el-Hossan in den Wad Draa mündet, und jetzt hatten wir wenigstens ununterbrochen glatten Weg." (LENZ 1884, Bd. 2, S. 8)

Die geplante Stelle für unsere Ankunft am Wadi Temenet (heute Tamanart [MichELIN 2009 , Nr. 742]) konnten wir nicht auf direktem Weg aus einer nordwestlichen Richtung erreichen. Denn etwa fünf Kilometer vor dem Wadi Tamanart war die in einem Tal 
gelegene, von uns befahrene steinige Piste durch Geröll in Folge eines oder mehrerer Unwetter großräumig versperrt. Nach einem Umweg von etwa 40 Kilometern erreichten wir das ersehnte Wadi Tamanart. Es bot sich ein grandioser Blick von der Höhe in das breite, sandige wasserlose Wadi. An den Berghängen kleben kleine Ortschaften. Wo es das Gelände zulässt, sind auf Terrassen vereinzelt landwirtschaftlich genutzte Flächen in der sonst kargen Landschaft angelegt.

\section{\# 66 Qasbah Temenet}

„... gegen 3 Uhr erweiterte sich plötzlich das Thal des Wad Temenet zu einer ausgedehnten Ebene, die Vegetation wurde reicher, Palmenwälder erschienen und wir erblickten uns zur Linken malerisch am Bergabhang liegend die Qasbah Temenet." (LENZ 1884, Bd. 2, S. 8)

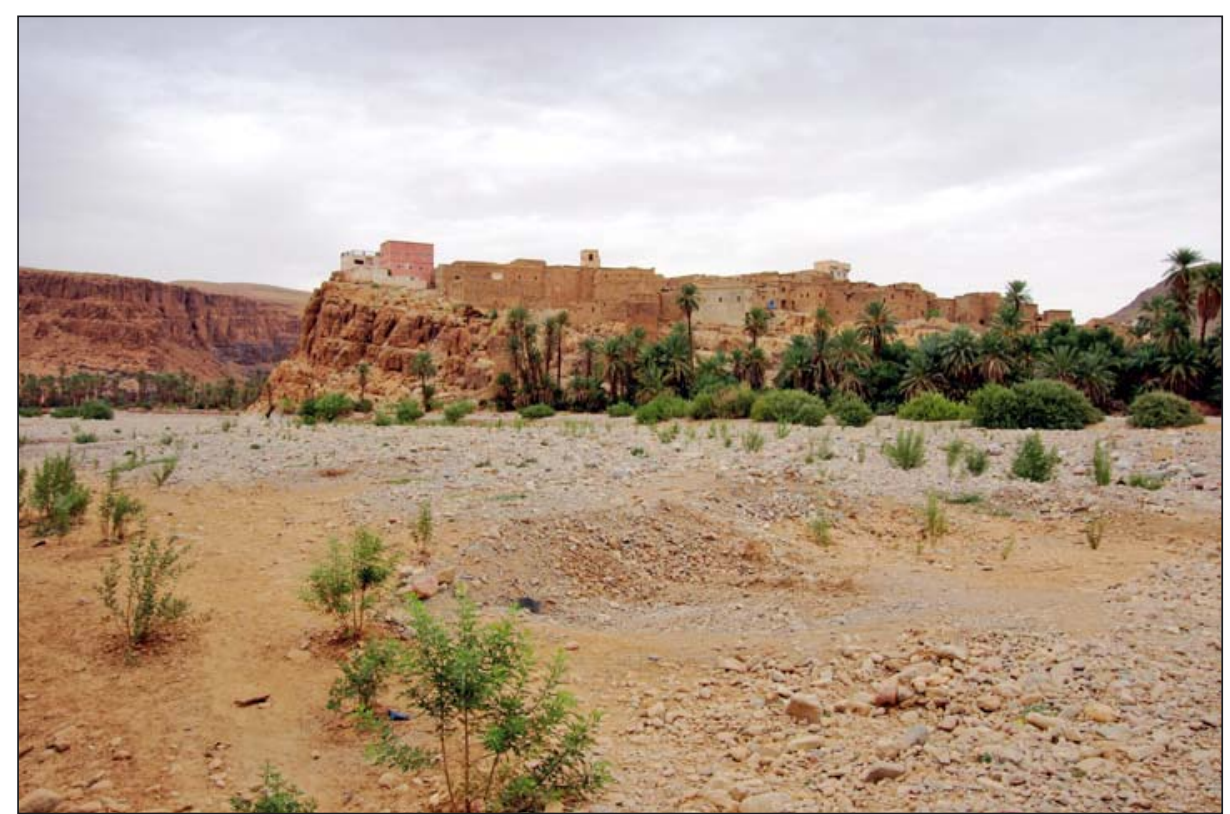

Abb. 16: Qasbah Tamanart

Am Westufer des Wadi Tamanart liegt heute der moderne Ort Tamanart. Wir querten den Ort und den dazugehörigen Palmenhain auf einem schmalen Weg und erreichten den alten Ort Tamanart auf dem linken Flussufer. Auf einem Felsrücken stehen intakte Häuser, die mehrheitlich aus Stampflehm gebaut und bewohnt sind.

\section{\# 69 westlich in die Berge}

„Von hier an verliessen wir die bisher eingeschlagene südliche Richtung und wandten uns wieder etwas westlich in die Berge." (LENZ 1884, Bd. 2, S. 9) 


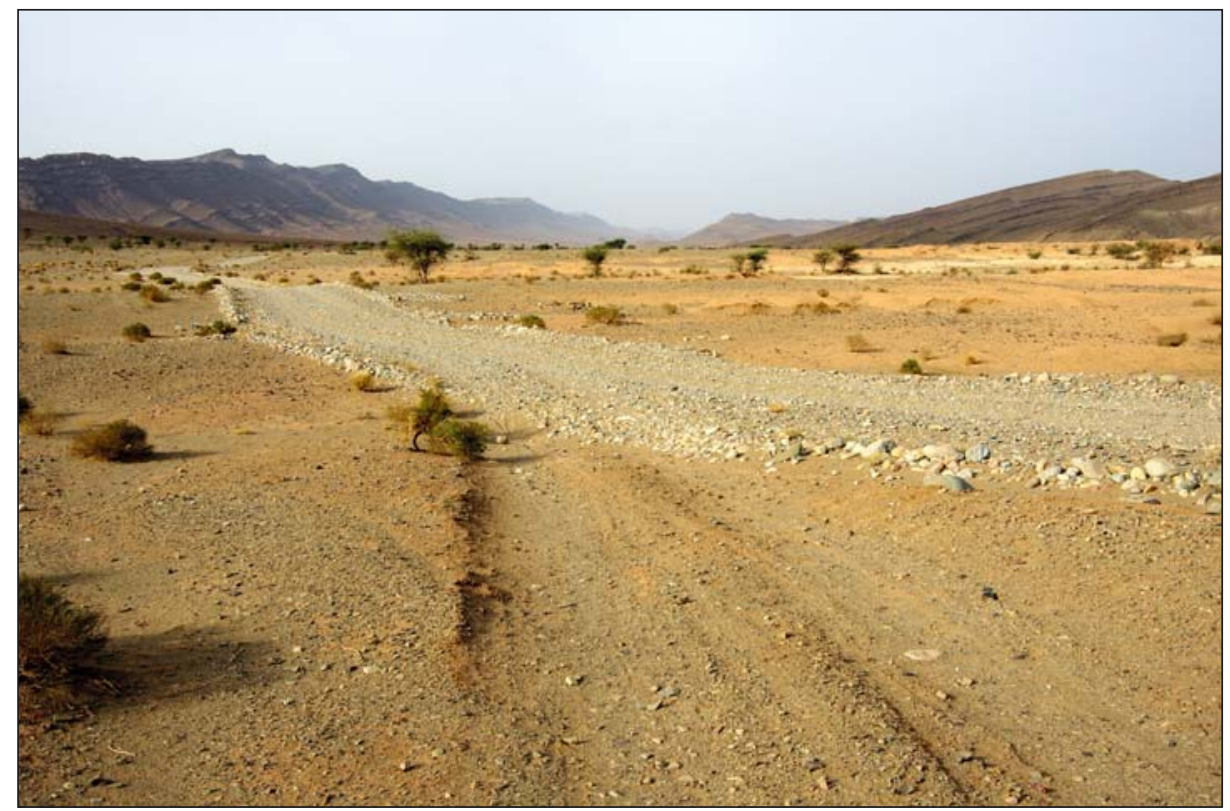

Abb. 17: westlich in die Berge

Nachdem sich das enge Tal des Tamanart geöffnet hat, führt auf einer Ebene eine gute Teerstraße zunächst parallel zum Wadi nach Süden. Auf dieser hätten wir das Ziel der Routenaufnahme, die Oase Foum El Hisn, bequem und schnell erreichen können. Aber auch wir verließen die südliche Richtung, um auf einer steilen Piste nach Westen in die Berge zu fahren. Auf diesem Weg konnten wir den Grund erkennen, warum zuvor von LENZ keine direkte südwestliche Richtung eingeschlagen wurde. Der direkte Weg wird versperrt durch ein mit tiefen Rinnen und Auswaschungen durchfurchtes Gebiet weichen Gesteines.

\section{\# 69.1 Quelle und Kanäle}

„... und kamen an eine Quelle mit reichlichem und köstlichem Wasser, an dem sich Mensch und Thier erquickte. Das Wasser wird in Kanäle gefasst und in die Palmengärten geführt." (LeNZ 1884, Bd. 2, S. 9)

Kurz vor Erreichen unseres Zieles erblickten wir am Berghang des östlichen Wadirandes eine offene, schmale Bewässerungsrinne. Diese führte vom Wasseraustritt der Quelle aus dem Berg heraus in die vor uns liegende Oase. Im kühlen, klaren Wasser tummelten sich Kaulquappen. 


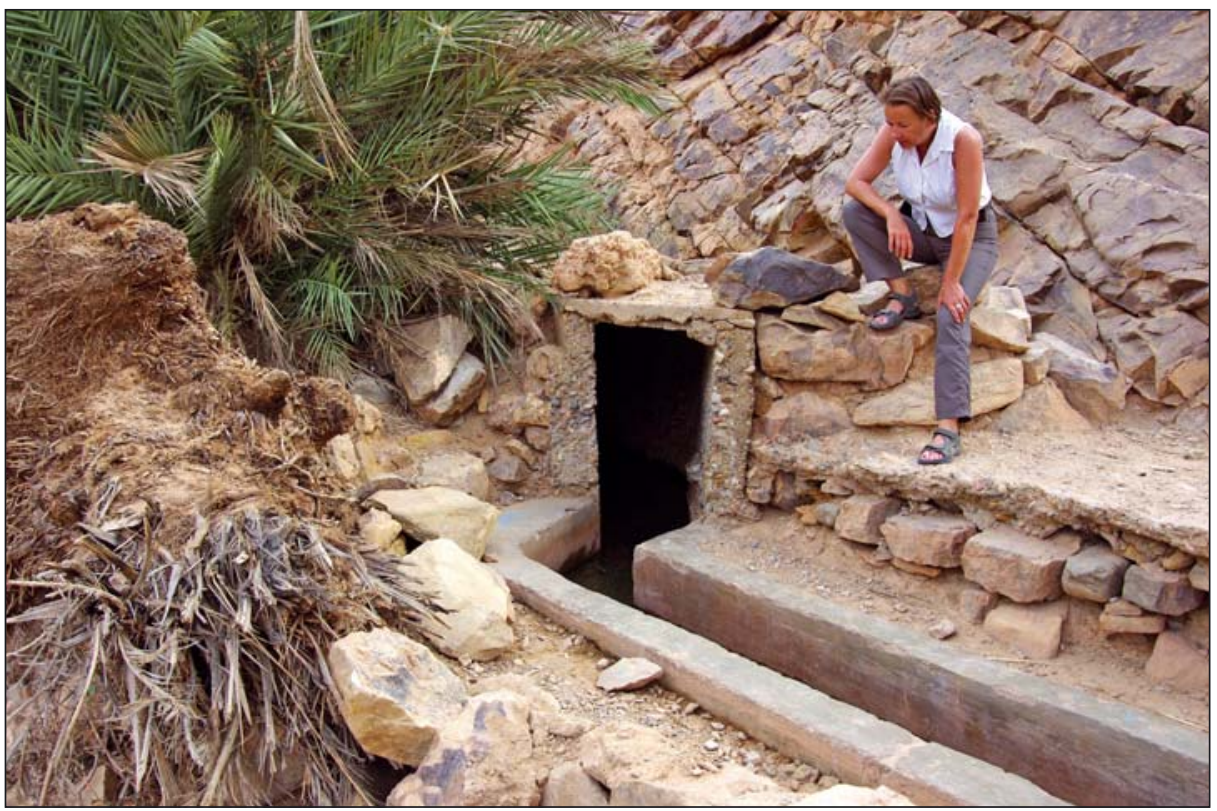

Abb. 18: Quelle und Kanal

\section{\#70 Fum el Hossan}

„Hier begegneten wir bereits wieder Menschen und etwas nach 5 Uhr zogen wir in die hübsch gelegene und gut gehaltene Stadt Fum-el-Hossan, auch Tizgi Ida Selam, oder Ait Selam genannt, ein, den Sitz des Scheichs der Araberkabyle Maribda. Hübsche, mit Mauern eingeschlossene Palmengärten vor der Stadt lockten mich an und ich hätte gern hier die Zelte aufgeschlagen, aber der grössern Sicherheit wegen bestimmte man, dass ich in der Stadt selbst, in einem Hause des Scheich Ali wohnen müsse. [...] Die Stadt Fum-el-Hossan liegt sehr schön dicht am Gebirge und ist von Palmengärten umgeben; man sieht von hier aus bereits in die gewaltige öde Hammada hinaus.

Die Bevölkerung mag einige tausend Seelen betragen, fast ausschliesslich Araber der Kabyle Maribda und deren Sklaven. Juden gibt es hier nicht. Die Stadt ist sehr gesund gelegen, das Wasser ist gut und reichlich vorhanden und die aus festgestampftem Lehm erbauten Häuser sind im allgemeinen nett und reinlich." (Lenz 1884, Bd. 2, S. 9)

Nach einer Woche Suchfahrt seit Marrakesch erreichten wir unser selbst gestecktes Ziel - die Oase Fum el Hossan (heute Foum El Hisn [Michelin 2009, Nr. 742]). Wir fuhren exakt durch das gleiche Tal nach Süden, durch das Oskar Lenz am 6. April 1880 marschierte. Beiderseits des Wadi Tamanart stehen an der Talöffnung unter Palmenhainen niedrige Häuser aus Stampflehm. Eine Straße querte das Wadi über eine Betonfurt. Etwa 500 Meter nach dem alten Ort erstreckt sich die neue, moderne Stadt Foum El Hisn. 


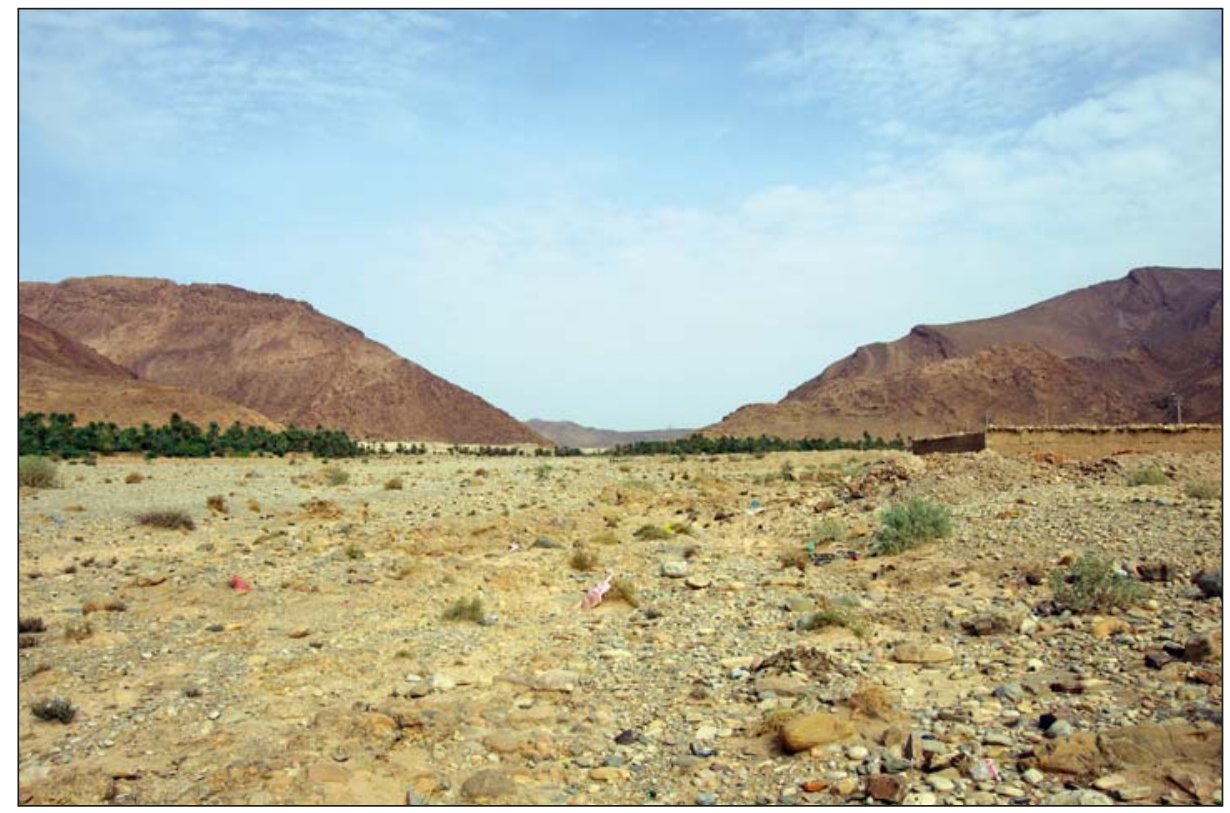

Abb. 19: Foum El Hisn von Süden 2011

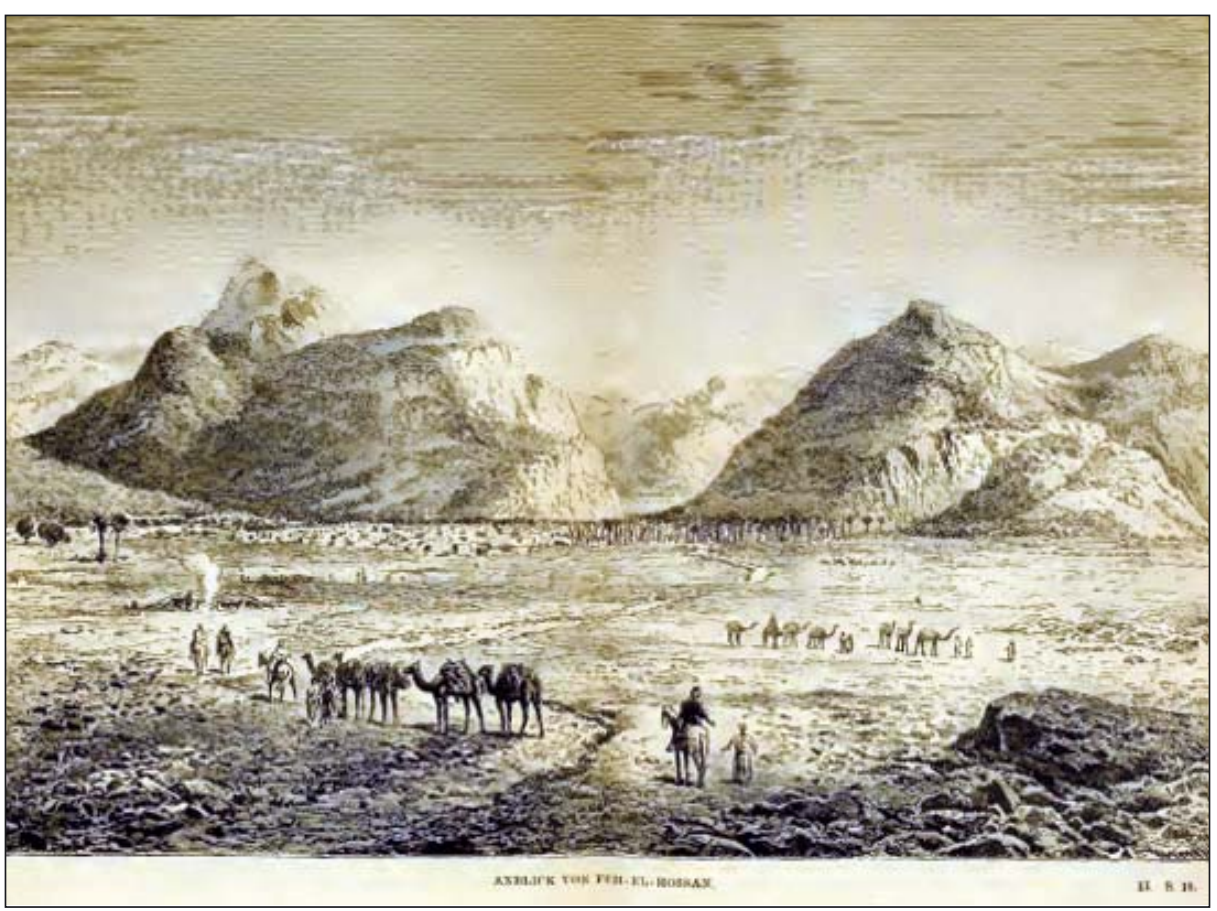

Abb. 20: Fum el Hossan von Süden 1880 (Lenz 1884, Bd. 2, S. 10-11) 


\begin{tabular}{|c|c|c|c|c|c|c|c|c|c|c|c|c|c|c|c|c|c|c|c|}
\hline 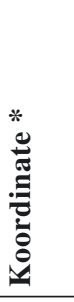 & 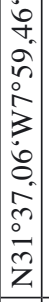 & 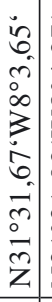 & 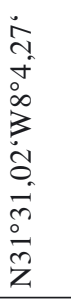 & $\begin{array}{l}0 \\
n \\
0 \\
0 \\
0 \\
0 \\
0 \\
8 \\
0 \\
0 \\
0 \\
0 \\
\\
z \\
\end{array}$ & 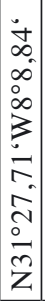 & 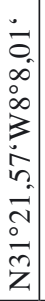 & 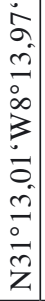 & 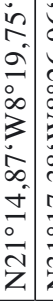 & 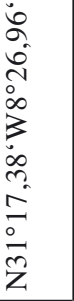 & $\mid \begin{array}{l}0 \\
0 \\
0 \\
0 \\
0 \\
0 \\
3 \\
0 \\
0 \\
0 \\
\tilde{n} \\
0 \\
0 \\
\bar{z} \\
z\end{array}$ & 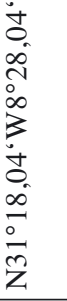 & 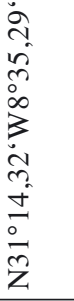 & 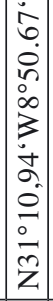 & 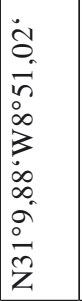 & 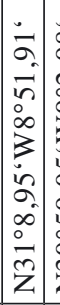 & 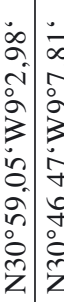 & 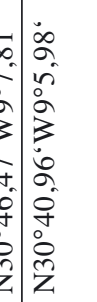 & 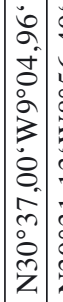 & 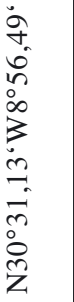 \\
\hline 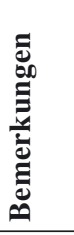 & & & & & & 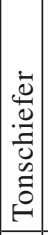 & & & & & 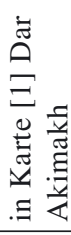 & & & 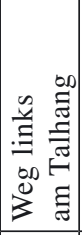 & & & & & \\
\hline 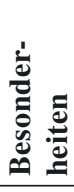 & & & & & & & & & & & & & & & 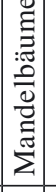 & & 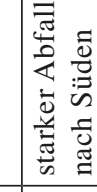 & & 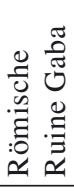 \\
\hline 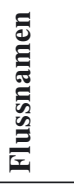 & & & 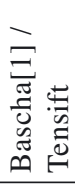 & & $\sum_{z}^{n}$ & & & & 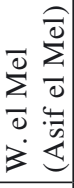 & & & & & & & & & & \\
\hline 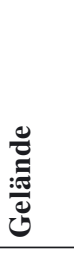 & 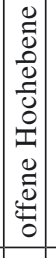 & & & & & 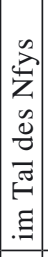 & & 离 & & & & & 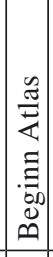 & 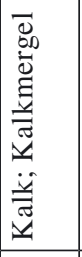 & 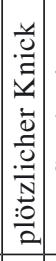 & 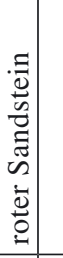 & & & \\
\hline 竞 & & & & & 章 & & & 3 & & $\mathrm{z}$ & & & & $n$ & 3 & & & & \\
\hline 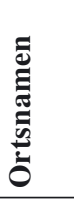 & 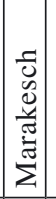 & & & 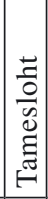 & & & 其 & & & & 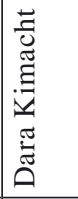 & 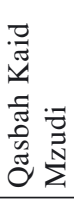 & 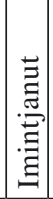 & & & & 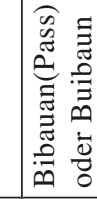 & 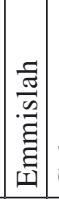 & $\begin{array}{l}\text { ల్ల్ } \\
\text { J్ }\end{array}$ \\
\hline Eٓ & \begin{tabular}{c}
0 \\
$\infty$ \\
$\infty$ \\
\hdashline \\
\\
0 \\
$\dot{0}$ \\
\end{tabular} & $\begin{array}{c}0 \\
\infty \\
\infty \\
-1 \\
0 \\
0 \\
\dot{0} \\
0\end{array}$ & \begin{tabular}{l}
0 \\
$\infty$ \\
$\infty$ \\
\hdashline \\
\\
0 \\
$\dot{0}$ \\
0
\end{tabular} & $\begin{array}{l}0 \\
\infty \\
\infty \\
0 \\
\dot{0} \\
\dot{8} \\
8 \\
\end{array}$ & $\begin{array}{l}0 \\
\infty \\
\infty \\
-1 \\
\dot{0} \\
0 \\
\dot{0} \\
\end{array}$ & $\begin{array}{c}0 \\
\infty \\
\infty \\
0 \\
\dot{0} \\
0 \\
\dot{0} \\
\end{array}$ & \begin{tabular}{c}
0 \\
$\infty$ \\
$\infty$ \\
\hdashline \\
0 \\
$\vdots$ \\
0 \\
0
\end{tabular} & 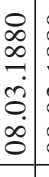 & 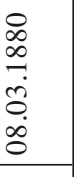 & $\begin{array}{c}0 \\
\infty \\
\infty \\
0 \\
\dot{\infty} \\
0 \\
\infty \\
0 \\
\end{array}$ & $\begin{array}{l}\infty \\
\infty \\
\infty \\
-1 \\
\\
0 \\
\infty \\
0 \\
0\end{array}$ & $\begin{array}{l}\infty \\
\infty \\
\infty \\
-1 \\
\dot{0} \\
0 \\
0 \\
0\end{array}$ & \begin{tabular}{l}
0 \\
$\infty$ \\
$\infty$ \\
\hdashline \\
$\dot{\sim}$ \\
0 \\
$=$ \\
$=$
\end{tabular} & $\begin{array}{l}\infty \\
\infty \\
\infty \\
\cdots \\
\dot{m} \\
0 \\
= \\
=\end{array}$ & \begin{tabular}{c}
$\infty$ \\
$\infty$ \\
$\infty$ \\
\hdashline \\
$\stackrel{2}{0}$ \\
$\stackrel{=}{=}$ \\
\end{tabular} & 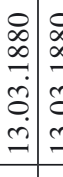 & 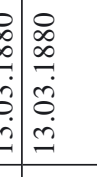 & \begin{tabular}{c}
0 \\
$\infty$ \\
$\infty$ \\
\hdashline \\
$\delta$ \\
0 \\
$\dot{J}$ \\
\end{tabular} & 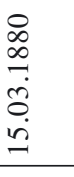 \\
\hline$\dot{\Xi} \dot{z}$ & 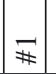 & \# & $\nRightarrow$ & $\nRightarrow$ & $\neq$ & * & $*$ & * & * & 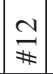 & $\#$ & * & * & $\#$ & 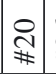 & 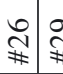 & $\vec{w}$ & 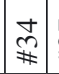 & \\
\hline
\end{tabular}




\begin{tabular}{|c|c|c|c|c|c|c|c|c|c|c|c|c|c|}
\hline 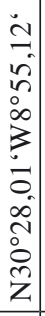 & 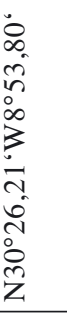 & 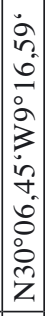 & 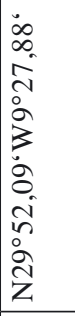 & 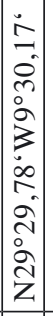 & 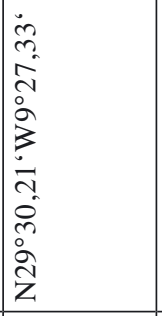 & 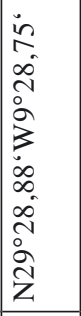 & 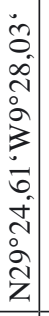 & 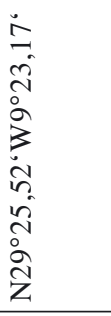 & 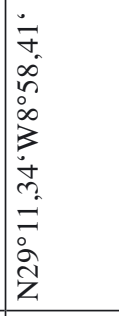 & 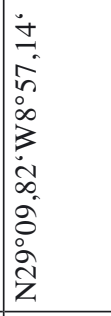 & 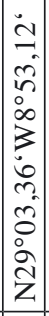 & 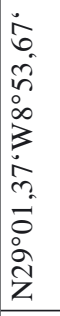 & 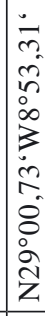 \\
\hline & 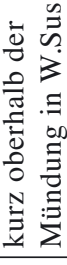 & & 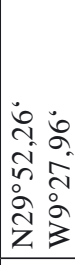 & & 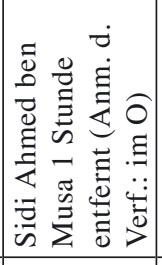 & 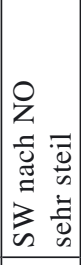 & & & 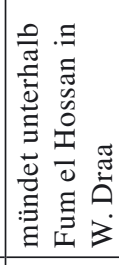 & 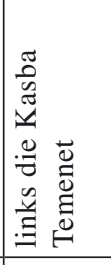 & & & \\
\hline & & & & & & & & 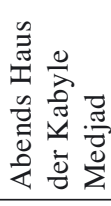 & $\begin{array}{l}3 \\
\vdots \\
\vdots \\
8 \\
\vdots \\
=\end{array}$ & 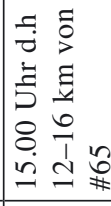 & & 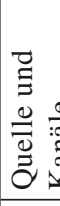 & \\
\hline & 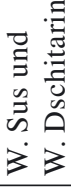 & & & & & & & & 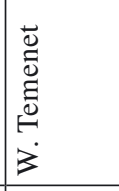 & & & & \\
\hline & & & & & & 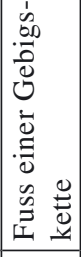 & 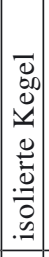 & & & 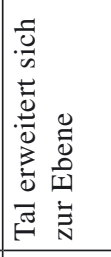 & \begin{tabular}{|c|c|} 
\\
$\infty$ \\
0 \\
$\infty$ \\
.$\Xi$ \\
.$\Xi$
\end{tabular} & & \\
\hline & & & & & & & & 0 & & & 3 & & \\
\hline 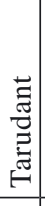 & & 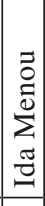 & 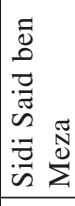 & 离 & & & & & & 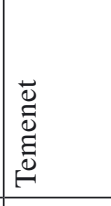 & & & 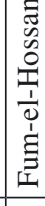 \\
\hline $\begin{array}{l}0 \\
\infty \\
\infty \\
\dot{3} \\
0 \\
\dot{n} \\
\end{array}$ & $\begin{array}{l}\infty \\
\infty \\
\infty \\
\dot{\infty} \\
\stackrel{d}{d} \\
\end{array}$ & 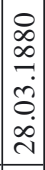 & $\begin{array}{l}\infty \\
\infty \\
\infty \\
-1 \\
0 \\
0 \\
\dot{\lambda} \\
\end{array}$ & $\begin{array}{c}0 \\
\infty \\
\infty \\
0 \\
\tilde{\infty} \\
0 \\
0 \\
n\end{array}$ & & $\mid \begin{array}{l}0 \\
\infty \\
0 \\
\dot{J} \\
0 \\
\dot{0} \\
0\end{array}$ & $\mid \begin{array}{l}0 \\
\infty \\
\infty \\
\dot{+} \\
\dot{+} \\
\dot{0} \\
\end{array}$ & $\begin{array}{l}\infty \\
\infty \\
\infty \\
\dot{0} \\
\dot{0} \\
\dot{0}\end{array}$ & \begin{tabular}{l}
$\infty$ \\
$\infty$ \\
$\infty$ \\
\hdashline \\
$\dot{0}$ \\
0 \\
0
\end{tabular} & \begin{tabular}{l}
$\infty$ \\
$\infty$ \\
$\infty$ \\
\hdashline \\
$\dot{0}$ \\
0 \\
0
\end{tabular} & 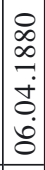 & $\begin{array}{l}0 \\
\infty \\
\infty \\
0 \\
\dot{0} \\
0 \\
\dot{0} \\
0\end{array}$ & $\begin{array}{l}0 \\
\infty \\
\infty \\
\dot{0} \\
0 \\
\dot{0}\end{array}$ \\
\hline 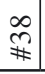 & $\underset{\$}{\$}$ & $\mid \begin{array}{l}0 \\
¥ \\
¥\end{array}$ & 夺 & $\mid \begin{array}{l}n \\
n \\
¥\end{array}$ & 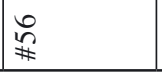 & $\mid \begin{array}{l}\infty \\
\cdots \\
¥\end{array}$ & 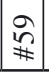 & 采 & 证 & $\begin{array}{l}8 \\
0 \\
\$\end{array}$ & $\mid \begin{array}{l}9 \\
\$\end{array}$ & 家 & $\underset{\#}{\stackrel{2}{2}}$ \\
\hline
\end{tabular}


Da Lenz in seinem Buch ein Bild der Oase mit Blick nach Norden abgedruckt hat, konnte etwa vom gleichen Aufnahmestandort und aus etwa der gleichen Perspektive ein Vergleichsbild aufgenommen werden. Der markante Taleinschnitt, die beiden Berghänge und die beiden Oasenteile sind mit guter Übereinstimmung zu erkennen. Allerdings sind im Bild von LENZ die Höhen der Berge deutlich erkennbar überzogen, was natürlich zu einem spektakuläreren Erscheinen des Ortes beiträgt. Dieser Unterschied zwischen zeitgenössischer Darstellung und der heutigen Realität ist auch schon bei Darstellungen anderer Reisender aus dieser Epoche auffällig und bekannt.

In der vorigen Datentabelle (vgl. Tab. 1) sind alle besuchten und zuvor beschriebenen Orte, weitere Informationen aus dem Reisewerk von LENZ sowie aktuelle Koordinaten aufgelistet.

\section{Fazit}

Der Reiseabschnitt zwischen Marrakesch und Taroudant konnte sehr gut durch eine Vielzahl von identifizierbaren Punkten nachvollzogen werden. Es zeigt sich, dass diese Wege heute überwiegend keine überregionalen Hauptverkehrswege sind, sondern eher regionale Bedeutung haben. Der von uns benutzte Übergang über den Kamm des Hohen Atlas hat heute nur eine lokale Verbindung und wurde erst vor kurzem asphaltiert.

Im Reiseabschnitt zwischen Taroudant und Fou el Hisn, also im Bereich des Antiatlas, konnten neben einigen sehr markanten Orten aber auch viele Ortsangaben von LENZ nicht zugeordnet werden. Auf diesem Abschnitt haben die heutigen Verkehrswege ebenfalls nur begrenzte lokale Bedeutung und liegen fernab bedeutender Fernverkehrswege. Insbesondere nordwestlich von Tamanart verlief die Route auf unbefestigten Pisten.

\section{Danksagung}

Mein besonderer Dank gilt meiner Frau, die mich bei der oft mühsamen Suchfahrt vor Ort unterstützt hat, die Navigation auf der berechneten Route erledigte und die Reiseaufzeichnungen im Routenbuch und im Reisetagebuch vornahm. Sehr dankbar bin ich Frau Annegret Werthmüller (Bern) für die Überlassung der alten Marokkokarten des Kartographen Erhard WERTHMÜLLER $(\dagger)$. Sie waren sehr hilfreich für die Planung der Reise. Ebenso möchte ich Herrn Dr. Brogiato (Leipzig) für seine freundliche Unterstützung danken. 


\section{Literaturverzeichnis}

Lenz O. (1884), Timbuktu - Reise durch Marokko, die Sahara und den Sudan. Leipzig, Brockhaus. Maroc. Direction de la Conservation foncière et des travauX topographiques (Hrsg.) (1944), Carte Maroc au 200.000, Tafraoute, LXXIX. Rabat.

Maroc. Direction de la Conservation foncière et des travaux topographiques (Hrsg.) (1946), Carte Maroc au 200.000, Argana, LXI. Rabat.

Maroc. Direction de la Conservation foncière et des travaux topographiques (Hrsg.) (1946), Carte Maroc au 200.000, Taroudant, LXX. Rabat 1946

Maroc. Direction de la Conservation foncière et des travaux topographiQues (Hrsg.) (1948), Carte Maroc au 200.000, Foum el Hassane, LXXXIX. Rabat.

Maroc. Direction de la Conservation foncière et des travauX topographiques (Hrsg.) (1949), Carte Maroc au 200.000, Chichaoua, LII. Rabat.

Maroc. Direction de la Conservation foncière et des travaux topographiques (Hrsg.) (1954), Carte Maroc au 200.000, Marrakech sud, LIII. Rabat.

Michelin (Hrsg.) (2009), Karte Marokko, 742. Paris

Neue Freie Presse, Wien, 3. März 1925, 21720.

Österreichische Akademie der Wissenschaften (ÖAW) (Hrsg.) (1970), Österreichisches Biographisches Lexikon 1815-1950, 5, 22. Wien, Verlag der Österreichischen Akademie der Wissenschaften.

ReIN J.J. (1887), Über Marokko. Berlin, Reimer.

WIKIPEDIA (Hrsg.) - http://de.wikipedia.org/wiki/Oskar_Lenz\#cite_note-.C3.96BL.7C5.7C140-3 